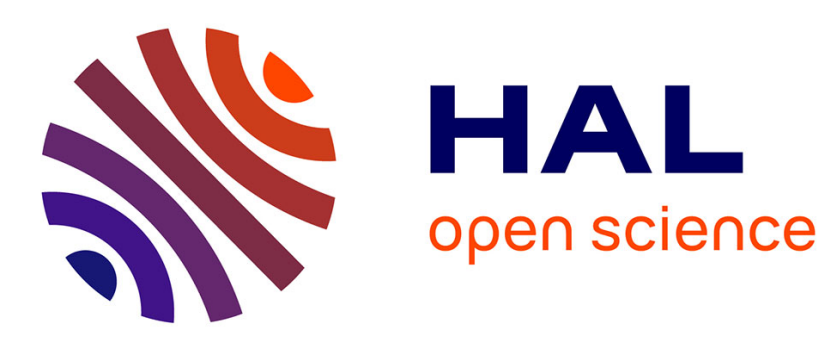

\title{
Some perspectives on the analysis and control of complementarity systems
}

Bernard Brogliato

\section{To cite this version:}

Bernard Brogliato. Some perspectives on the analysis and control of complementarity systems. IEEE Transactions on Automatic Control, 2003, 48 (6), pp.918-935. 10.1109/TAC.2003.812777. hal01660014

\section{HAL Id: hal-01660014 https://hal.science/hal-01660014}

Submitted on 9 Dec 2017

HAL is a multi-disciplinary open access archive for the deposit and dissemination of scientific research documents, whether they are published or not. The documents may come from teaching and research institutions in France or abroad, or from public or private research centers.
L'archive ouverte pluridisciplinaire HAL, est destinée au dépôt et à la diffusion de documents scientifiques de niveau recherche, publiés ou non, émanant des établissements d'enseignement et de recherche français ou étrangers, des laboratoires publics ou privés. 


\title{
Some Perspectives on the Analysis and Control of Complementarity Systems
}

\author{
Bernard Brogliato
}

\begin{abstract}
This paper is devoted to presenting controllability and stabilizability issues associated to a class of nonsmooth dynamical systems, namely complementarity dynamical systems. The main existing results are summarized, and some possible research directions are provided. Convex analysis and complementarity problems are claimed to be the main analysis tools for control related studies. This paper mainly focuses on mechanical applications.
\end{abstract}

Index Terms-Complementarity, control, convex analysis, hybrid dynamics, measure differential inclusions, mechanical systems, nonsmooth, stability.

\section{NOMENCLATURE}

DI Differential inclusion.

MDI Measure DI.

AC Absolutely continuous.

RCLBV Right-continuous of local bounded variation.

CP Complementarity problem.

CS Complementarity system.

ACS Affine complementarity system.

LCS Linear complementarity system.

LCP Linear complementarity problem.

DES Discrete-event system.

VI Variational inequality.

\section{INTRODUCTION}

$\mathbf{J}$ . J. MOREAU made fundamental contributions to convex analysis and nonsmooth mechanics [64]-[73], which have had considerable influence in several branches of mechanics (discrete systems, fluid mechanics, elasto-plasticity, and friction) [53], [79], [39], [63], and for the numerical simulation of nonsmooth mechanical systems [15]. The reader is referred to [25] for an introduction to nonsmooth mechanics and to [82] for a complete panorama of the topic in applied mathematics and mechanics. In addition, nonsmooth analysis has made significant progress and has allowed researchers to solve important problems like in optimal control and calculation [100], [74], by introducing tools like Clarke's generalized gradient [79], [23], nonsmooth versions of Newton's algorithms [2], and complementarity problems [8], [74]. In this context, it is worth recalling that nonsmoothness may not only arise at the modeling level, but may also be necessary to solve some

The author is with INRIA Rhône-Alpes, ZIRST Montbonnot, 38334 Saint Ismier Cedex, France (e-mail: Bernard.Brogliato@inrialpes.fr). abstract problems. For instance minimum-time optimal control yields cost functions which are both nondifferentiable and nonconvex. Recently, mechanical systems subject to inequality (or unilateral) constraints have been the object of renewed interest in the mechanical engineering and applied mathematics scientific communities; see [12], [14], [33], [35], [51], [63], [81], and [96]. In parallel, the analysis and control of hybrid dynamical systems has become an active investigation area in the systems and control scientific community [47], [3], [31], [48], [97], [87]. In particular, complementarity systems are an interesting class of hybrid dynamical systems [20], [40]-[42], [89], [88]. Roughly speaking, complementarity systems consist of a dynamical system that is coupled to a set of algebraic conditions through a Lagrange multiplier (also called a slack variable in nonlinear programming). They can also be seen as dynamical systems subject to a particular type of generalized nonsmooth constraint. Complementarity dynamical systems find applications in mechanical systems (multibody systemsmanipulators, bipedal robots [46], controlled structures, tethered satellites [52], haptically augmented teleoperation, part feeding, automatic assembly, material handling systems, etc., structural mechanics, elastohydrodynamics lubrication, liquid slosh phenomena, collisions of fluids and solids, phase changes, etc. [33]), electrical circuits (nonlinear circuits with diodes, MOS transistors, operational amplifiers [41], [99]), control theory (optimal control with state constraints, model predictive control [7], variable structure systems [87]), traffic and oligopolistic market equilibrium problems [8], [75], economics (production, comsumption, the theory of option pricing, Walrasian problems [32]), models for biological systems (genetic networks, bacteria growth, gene regulatory networks that describe the regulatory interactions between genes and gene products in a cell [49]), etc. This paper is devoted to the controllability and the stabilizability of complementarity dynamical systems. Similarly to the fact that nonsmooth analysis is not a straightforward extension of classical analysis [23], the control of such nonsmooth dynamical systems is by far not a direct extension of the control of smooth systems. The same can be asserted to numerical analysis and simulation [1], [15] and bifurcation analysis [55]. This paper does not pretend to survey the wide area of nonsmooth systems. For more complete bibliographies, the reader is referred to [12] and [15]. It rather concentrates on a specific class and mainly aims at pointing out some open problems (marked by $\mathbf{O P}^{1}$ ) and the main features of the analyzed systems. The paper is organized as follows: In

\footnotetext{
${ }^{1}$ Some of these open problems may be very challenging, and some others more trivial. It is rather difficult to classify them at this stage. Other research directions can be found in [41] and [15].
} 
Section II the dynamics of complementarity systems is presented. Several typical examples are given in Section III, and the link with various nonsmooth dynamical systems and differential inclusions is made in Section IV. Section V is devoted to controllability issues, while Section VI focuses on stability and stabilization. Conclusions end the paper, and some technical informations are provided in Appendices A and B. Convex analysis, complementarity problems, generalized equations, impact Poincaré maps, measure differential inclusions, are advocated to be important analysis tools. Though fundamental topics in control, observability (see [61]) and identification problems are not discussed. Mechanical systems with unilateral constraints and friction are ubiquitous in everyday life and in industry. Moreover, mechanics has always had a leading role in Science, and many results motivated by Mechanics have found applications or extensions in other scientific fields. The main focus of this paper is, therefore, on mechanical systems, despite many other systems may be modeled in a complementarity framework (like electrical networks) as the aforementioned list shows.

\section{COMPlementary DynAmical Systems}

\section{A. General Dynamics}

The class of finite-dimensional controlled nonsmooth dynamical systems on which we will focus in this paper can be represented by the following set of differential and algebraic equations and conditions:

$$
\left\{\begin{array}{l}
\dot{x}=f(x, t, u, \lambda), \text { almost everywhere, } x\left(\tau_{0}\right)=x_{0} \\
z=\zeta(x, u, \lambda) \\
\mathcal{C}^{\star} \ni y \perp \Lambda(\lambda) \in \mathcal{C} \\
g(y, \lambda, u, x, t)=0 \\
\text { State } x(\cdot), \text { reinitialization rule }
\end{array}\right.
$$

where $x \in \mathbb{R}^{n}$ is the state of the continuous dynamics, $t \geq \tau_{0}$ is a real number, $z$ is some measurable output signal available for feedback, $u \in \mathbb{R}^{l}$ is a control signal to be chosen in some admissible set $\mathcal{U}$, the slack variable $\lambda \in \mathbb{R}^{m}$ and the signal $y \in \mathbb{R}^{m}$ constitute a pair of complementarity variables as indicated in the third line of $(1)$, where $\Lambda(\cdot)$ is some function. The symbol $\perp$ means that $y$ and $\Lambda(\lambda)$ have to be orthogonal. The sets $\mathcal{C}^{\star}$ and $\mathcal{C}=\left\{z \mid z^{T} v \leq 0, \forall v \in \mathcal{C}^{\star}\right\}$ are a pair of polar convex cones, $\tau_{0}$ is the initial time. Let us note that the first equality in (1) will generally be satisfied only almost everywhere in the Lebesgue's measure sense, because the solution $x(\cdot)$ of (1) will generally not be differentiable everywhere. Following [89], dynamical systems as in (1) may be named complementarity systems [CS, or systems subject to generalized constraints, made of the third and fourth lines in (1)]. In order to integrate the dynamical part of (1) (i.e., the first line), one needs to calculate $\lambda$. It will be shown on several examples how the slack variables $\lambda_{i}, i \in\{1, \ldots, m\}$ are calculated at each time $t$. Finally a state reinitialization rule will generally be needed to integrate trajectories on a time interval of strictly positive measure. The interplay between the various ingredients in (1) may be rather complicated, and this is what makes the study of such controlled dynamics challenging. Before examining some simple examples, let us briefly introduce complementarity problems (see more in Appendix B).

Complementarity conditions have been introduced by Moreau [64] in contact mechanics, and Karush et al. in quadratic programming [74]. They yield CP of the form $V_{1} \ni y \perp z \in V_{2}$ : the $m$-dimensional vectors $y$ and $z$ have to belong to the sets $V_{1}$ and $V_{2}$, respectively, and are orthogonal. If $V_{1}$ and $V_{2}$ are a pair of polar cones, then $V_{1} \ni y \perp z \in V_{2}$ is a Cone CP [8]. If $y=A z+B$ for some matrix $A$ and vector $B$ and if $V_{1}=V_{2}=\left(\mathbb{R}^{+}\right)^{m}$, then $0 \leq y \perp z \geq 0$ is equivalent to the Linear $C P(L C P) z \geq 0, A z+B \geq 0, z^{\bar{T}}(A z+B)=0$, denoted as $\operatorname{LCP}(z)$. A is the LCP matrix. If the matrix $A$ is a P-matrix (i.e., all its principal minors are positive), then the $\operatorname{LCP}(z)$ has a unique solution independently of $B$ [74]. If $y=g(z)$ for some nonlinear function $g(\cdot)$, then $0 \leq y \perp z \geq 0$ is a nonlinear $C P(N C P)$.

When they exist, the left and right-limits of a function $f(\cdot)$ at $t$ are denoted as $f\left(t^{-}\right)$and $f\left(t^{+}\right)$, respectively.

Example 1: Let us consider the following complementarity systems:

$$
\begin{aligned}
& \left(S_{1}\right)\left\{\begin{array}{l}
\dot{x}(t)=-x(t)+\alpha \lambda-a \\
0 \leq \lambda \perp y=x(t) \geq 0
\end{array}\right. \\
& \left(S_{2}\right)\left\{\begin{array}{l}
\dot{x}(t)=-x(t)+\lambda \\
0 \leq \lambda \perp y=x(t)+\alpha \lambda \geq 0
\end{array}\right. \\
& \left(S_{3}\right)\left\{\begin{array}{l}
\xi^{(3)}(t)=\lambda-a \\
0 \leq \lambda \perp y=\xi(t) \geq 0
\end{array}\right.
\end{aligned}
$$

where $a>0$ is a constant and $\alpha=1$ or $\alpha=-1$. Consider $\left(S_{1}\right)$, and let $x\left(\tau_{0}\right)>0$. Thus, initially $\lambda=0$ so that $x(\cdot)$ decreases toward 0 in finite time $t_{0,1}$, i.e., $x\left(t_{0,1}\right)=0$. Notice that due to the complementarity conditions, one has $\lambda=0$ on $\left[\tau_{0}, t_{0,1}\right)$. In order to continue the integration for $t \geq t_{0,1}$, there must exist at least one $\lambda$ such that both the differential equation and the complementarity conditions are simultaneously satisfied. Let us propose $\lambda(t)=(x(t)+a) / \alpha$. Then, $\dot{x}(t)=0$ so that on $\left(t_{0,1},+\infty\right)$ one has $x(t)=0$. One notices that if $\alpha=1$ then $\lambda(t)=a / \alpha>0$, however if $\alpha=-1$ then $\lambda(t)<0$ which is impossible. Notice that in the latter case one has $\dot{x}\left(t_{0,1}^{+}\right)=-\lambda-a<0$, consequently if $\alpha=-1$, there is no nonnegative $\lambda$ which allows to satisfy $x \geq 0$ on an interval $\left(t_{0,1}, t_{0,1}+\epsilon\right)$, for any $\epsilon>0$. Notice also that we have written the right-limit for $\dot{x}(\cdot)$ at $t_{0,1}$ because the solution we are looking for is indeed not differentiable at $t_{0,1}$ since $\dot{x}\left(t_{0,1}^{-}\right)=-a$ whereas $\dot{x}\left(t_{0,1}^{+}\right)=0$. Thus, if $\alpha=-1$, the integration cannot be continued after $t_{0,1}$. If $\alpha=1$ the previous choice allows one to integrate over $\left[\tau_{0},+\infty\right)$. Moreover, it can be shown that this is the only possible solution. Actually the proposed choice for $\lambda$ comes from the following: assume that $x(t)=0$ on a nonzero time interval. Then $(0 \leq \lambda \perp y=$ $x(t) \geq 0) \Longrightarrow(0 \leq \lambda \perp \dot{x}(t) \geq 0)$, from which it follows that $\lambda$ is the solution of the LCP $0 \leq \lambda \perp \lambda-a \geq 0$. This LCP always possesses a unique solution which is precisely the previous one. And if $\alpha=-1$ the corresponding LCP has no solution. Therefore, one sees that once the boundary $x=0$ has been reached, we have been able to exhibit a new slack variable $\lambda$ such that integration of $x(\cdot)$ can be continued, and the 
complementarity conditions remain satisfied provided the LCP matrix $\alpha$ (which is here a scalar) is positive. Assume now that at time $t_{1,2}>t_{0,1}$ the vector field switches to $-x(t)+a$ and is right-continuous at $t_{1,2}$. Then one looks for the solution of the same LCP which gives $\lambda\left(t_{1,2}^{+}\right)=0$. Thus, $\dot{x}\left(t_{1,2}^{+}\right)=a>0$ so that $x(\cdot)$ leaves the surface $x=0$ at $t_{1,2}$ and reenters the domain $\{x \mid x>0\}$.

Consider now $\left(S_{2}\right)$. This time the complementarity conditions directly yield a LCP for the slack variable $\lambda$. If $\alpha=-1$ then this LCP has no solution for any $x\left(\tau_{0}\right)<0$. It has the two solutions $\lambda=x$ or $\lambda=0$ for $x\left(\tau_{0}\right) \geq 0$ (see, e.g., [12, fig. 5.19]). Consequently in this case there are two solutions $x(\cdot)$ emanating from $x\left(\tau_{0}\right)$ and at each time $t$ such that $x(t)>0$, and uniqueness fails. Let $\alpha=1$. If $x\left(\tau_{0}\right) \leq 0$ the unique solution of the LCP is $\lambda\left(\tau_{0}\right)=-x\left(\tau_{0}\right) \geq 0$. Then, $x(\cdot)$ increases toward 0 exponentially on $\left[\tau_{0},+\infty\right)$ with $\lambda=-x(t)$. If $x\left(\tau_{0}\right)>0$ then $\lambda\left(\tau_{0}\right)=0$ is the unique solution of the LCP and $x(\cdot)$ decreases to 0 exponentially on $\left[\tau_{0},+\infty\right)$ with $\lambda=0$.

In both $\left(S_{1}\right)$ and $\left(S_{2}\right)$ with $\alpha=1$, we have exhibited a unique continuous solution that is almost everywhere differentiable for $\left(S_{1}\right)$ and everywhere differentiable for $\left(S_{2}\right)$. Consider now $\left(S_{3}\right)$. If $\xi\left(\tau_{0}\right)>0, \dot{\xi}\left(\tau_{0}\right)=\ddot{\xi}\left(\tau_{0}\right)=0$, then on a finite time interval $\left[\tau_{0}, t_{0}\right), \xi(t)$ decreases toward $\xi\left(t_{0}\right)=0$, and $\lambda(t)=0$. One has $\dot{\xi}\left(t_{0}^{-}\right)=-(a / 2)\left(t_{0}-\tau_{0}\right)^{2}<0$, $\ddot{\xi}\left(t_{0}^{-}\right)=-a\left(t_{0}-\tau_{0}\right)<0$. Clearly, at $t_{0}$, the value of $\lambda$ has to change in order to keep $\xi(\cdot)$ nonnegative. Even more, $\xi(t) \geq 0$ on $\left(t_{0}, t_{0}+\epsilon\right)$ for some $\epsilon>0$, implies that both $\dot{\xi}(\cdot)$ and $\ddot{\xi}(\cdot)$ jump to nonnegative values at $t_{0}$. This implies that $\lambda\left(t_{0}\right)$ cannot be a function, but must be a distribution involving both the Dirac distribution $\delta_{t_{0}}$ and its derivative $\dot{\delta}_{t_{0}}$. At this stage, one needs some rule to perform the calculation of the jumps in $\dot{\xi}(\cdot)$ and $\ddot{\xi}(\cdot)$. If this rule says that $\dot{\xi}\left(t_{0}^{+}\right)=\ddot{\xi}\left(t_{0}^{+}\right)=0$ then the integration can continue on $\left(t_{0},+\infty\right)$ with $\lambda=a$, so that $\xi(t)=\dot{\xi}(t)=\ddot{\xi}(t)=0$ on $\left(t_{0},+\infty\right)$. Using the fact that $\xi(t)=\dot{\xi}(t)=\ddot{\xi}(t)=0$ on a nonzero time interval together with $0 \leq \lambda \perp \xi \geq 0$, we conclude that $0 \leq \lambda \perp \xi^{(3)} \geq 0$ holds. Consequently $\lambda=a$ is calculated at time $t$ as the unique solution of this LCP. If the rule states that $\dot{\xi}\left(t_{0}^{+}\right)>0, \ddot{\xi}\left(t_{0}^{+}\right)>0$, then $\xi(\cdot)$ bounces on the boundary $\xi=0$ at $t_{0}$ and $\xi(t)>0$ on $\left(t_{0}, t_{0}+\epsilon\right)$ for some $\epsilon>0$.

These examples do not encapsulate all the features of complementarity systems and are not meant to be rigorous proofs for existence and uniqueness of solutions. However they do illustrate some basic peculiarities. One notices that the vector field remains continuous in $x(\cdot)$, until some boundary is reached by the state $x(\cdot)$. Then the multiplier $\lambda$ is calculated and modifies the vector field of the continuous dynamics, in such a way that the integration of $x(\cdot)$ proceeds. It is noteworthy that the leading Markov parameter [93] of the system with output $y$ and input $\lambda$ plays a significant role in the analysis and that it is closely related to the existence and uniqueness of solutions of some LCP constructed from the complementarity conditions and the dynamics. The well-posedness results in [20], [40], [42], [89], and [88] are based on this observation. When $\dot{x}=A x+E \lambda, y=C x+D \lambda, \Lambda(\lambda)=-\lambda$ and $\mathcal{C}^{\star}=\mathbb{R}^{+},(1)$ is named a LCS. LCS have been presented for the first time in [89]. If $D$ is a $P$ matrix then $\lambda$ is the unique solution of the LCP $0 \leq \lambda \perp C x(t)+D \lambda \geq 0$ at each $t$.
A particular feature of CS compared to other classes of hybrid systems (see [3], [47], and [97]), is that CP and convex analysis are at the core of their analysis. This is clear for both the mathematical [87], [42], [40] and numerical [15] aspects. One objective of this paper is to prove that this is also the case for control and systems analysis. As we shall see later, such systems can also be considered as differential inclusions. Models as in (1) are quite interesting because they are not too general, but yet are rich enough to potentially present a lot of challenging problems to systems and control theorists. Moreover, the engineering applications are numerous. Let us notice that the discrete-time counterpart to (1) exists [7], [43], but we shall focus on the continuous-time case only.

If $z$ is a solution of this NCP, then it is also a zero of the nonsmooth complementarity function $\bar{g}(z)=\min [z, g(z)]$. The set of piecewise linear equations $\bar{g}(z)=0$ is equivalent to $0 \leq$ $g(z) \perp z \geq 0$ [8]. Other complementarity functions exist. The constraint $\bar{g}(z)=0$ may be named a generalized constraint.

Remark 1: If $\mathcal{C}^{\star}=\mathbb{R}^{+}, \Lambda(\lambda)=-\lambda$, and if $y=$ $h(x, t, u, \lambda)$ it follows that the third and fourth lines of (1) can be rewritten as $\bar{g}(x, t, u, \lambda)=0$ for some complementarity function $\bar{g}$. Thus, the dynamics in (1) may be seen as a nonsmooth differential algebraic equation in this case.

\section{B. Associated Hybrid Automaton}

The time-integration of the systems in (2) suggests that there is a natural connection between the dynamics in (1) and so-called hybrid dynamical systems, i.e., dynamical systems whose evolution is the result of the interplay between continuous and discrete-event dynamics [87], [41]. In (1), the continuous part is in the first two lines, the third and fourth lines describe the discrete-event part (the modes and the conditions for transitions between modes to occur). The last line describes how transitions between modes occur. The state of the system, therefore, consists of a variable that takes its values in a continuous space, and variables that take their value in a finite set $L$, i.e., $\operatorname{card}(L)<+\infty$. The DES state for CS as in (1) can be in a first instance constructed from the modes that correspond to the values of the variables $y_{i}, i \in\{1, \ldots, m\}$. Let us consider the case of a LCS. For each component $y_{i}$, the complementarity conditions yield two modes: $y_{i}=0$ or $y_{i}>0$. Hence, one has a priori $\operatorname{card}(L)=2^{m}$. The events (or transitions) correspond to trajectories passing from one mode to another one, implying a change in the structure of the vector field $f(x, t, u, \lambda)$. In $\left(S_{1}\right)$ there are such events at times $t_{0,1}$ and $t_{1,2}$, and in $\left(S_{3}\right)$ at time $t_{0}$.

Let us denote the states of the DES part as $S_{i} \in L, 1 \leq$ $i \leq \operatorname{card}(L)$, and a trajectory (also named a path) as $\mathcal{S}$. A path thus consists of a finite number of DES states $S_{i}$ together with transition rules which allow the system to evolve from one state $S_{i}$ to another state $S_{j}$. One may consider that a mode is active, if and only if $x(\cdot)$ has remained during a time interval of positive Lebesgue measure in this mode. The reinitialization of the state implies a discontinuity in $x(\cdot)$. In general, the activation of a new mode cannot be done without a reinitialization of the state $x(t)$, hence, the fifth line in (1). See, e.g., $\left(S_{3}\right)$ where the state 
has to jump at $t_{0}$ to continue the integration. However, in some cases a nondifferentiable state $x(t)$ may suffice for a trajectory to pass from one mode to another one, see examples $\left(S_{1}\right)$ and $\left(S_{2}\right)$ in (2). Transitions may even occur smoothly, as in the case of a mechanical system if the constraint is attained tangentially in the phase space.

Generically event times which correspond to a trajectory passing from the mode $S_{i}$ to the mode $S_{j}$ are denoted as $t_{i, j}$, and reinitialization times are denoted as $t_{k}, k \geq 0$.

The complexity of CS is further illustrated by simple mechanical systems, where an infinity of events can occur in finite time (finite accumulation of impact times, i.e., a particular type Zeno behavior [87, p. 10]), and several such finite accumulations may be repeated (possibly an infinity of times as $t \rightarrow+\infty$ ). A well-known example is the bouncing-ball dynamics $\ddot{q}=-g+\lambda$, $q(0)>0, \dot{q}(0)<0,0 \leq \lambda \perp q \geq 0, \dot{q}\left(t_{k}^{+}\right)=-e \dot{q}\left(t_{k}^{-}\right)$, $e \in(0,1)$ and $\lim _{k \rightarrow \infty} t_{k}=t_{\infty}<+\infty$. There is a sequence of impact times $\left\{t_{k}\right\}_{k \geq 0}$, that is followed by a switch from mode $q>0$ to mode $q=0$ at time $t_{0,1}=t_{\infty}$ [notice that $\dot{q}\left(t_{\infty}^{+}\right)=\dot{q}\left(t_{\infty}^{-}\right)=0$ so that this transition occurs without velocity jump]. Consequently on $\left[0, t_{\infty}\right)$ the system evolves almost all the time in the mode $q>0$. Obviously, in some cases, a time $t_{k}$ is also a mode switching time (see $\left(S_{3}\right)$, and plastic impacts in mechanics).

The distinction between the times $t_{i, j}$ and $t_{k}$ is not necessary from a general hybrid dynamical systems point of view, because they can all be embedded into transition or event times [87, Chs. 1 and 2]. However, it may sometimes be convenient to distinguish between state jumps and variations of the structure of vector fields since they may not have the same consequences on control properties.

Remark 2: The associated automaton has no discrete control. In other words the transitions between the modes $S_{i}$ as previously defined, are only function of the continuous part of the system. This may have important consequences on control properties.

In this paper, we will not provide many details on the well-posedness problems [existence and uniqueness of solutions $x(t)=\varphi\left(t ; \tau_{0}, x_{0}, u\right)$ for all $t \geq \tau_{0}$ and in a suitable function space]. Examples in (2) provide a rapid overview for some CS.

Assumption 1: There exists $x_{0}$ and $u:\left[\tau_{0}, T\right] \rightarrow \mathbb{R}^{l}$ such that (1) possesses a unique solution $x(t)=\varphi\left(t ; x_{0}, u, \tau_{0}\right)$ on $\left[\tau_{0}, \tau_{0}+\varepsilon\right)$ for some $\varepsilon>0, \forall \tau_{0} \geq 0, T>\tau_{0}+\varepsilon$. Depending on the ingredients in (1), solutions may be of different nature (AC, RCLBV, piecewise continuous, etc.). The least requirement is that solutions possess a right limit everywhere.

In other words assumption 1 states that there exist an initial state and a control input (possibly $u \equiv 0$ ) such that (1) can be integrated on a nonzero time-interval. Otherwise we consider that the model can be rejected. Clearly the space in which the solution lives, may influence control studies. In particular the input $u(\cdot)$ has to take its values in a suitable space $\mathcal{U}$, and this has consequences on controllability. Also notice that assumption 1 includes possible initial state jumps and that the solutions may be discontinuous with respect to initial data $x_{0}$. Such evolution problems may be named prospective [73], because at each $t$ one looks for right limits of the solution.

\section{Basic Notions From Convex Analysis}

The following notions [44], [65], [86] are fundamental tools for the analysis of complementarity systems, and we will use most of them. Let $K$ denote a closed convex set. Its indicator function is defined as $\psi_{K}(x)=0$ if $x \in K$ and $\psi_{K}(x)=+\infty$ if $x \notin K$. The subdifferential of a lower semicontinuous (lsc) convex function $f(\cdot)$ at $y$ is denoted as $\partial f(y)$ and is the set of subgradients, i.e., vectors $\gamma$ satisfying $f(x)-f(y) \geq \gamma^{T}(x-y)$ for all $x$. The subdifferential replaces the derivative, for nonsmooth convex functions. If $f(x)=+\infty$ for $x<0, f(x)=x$ for $x \geq 0, x \in \mathbb{R}$, then $f(\cdot)$ is convex lsc and $\partial f(0)=(-\infty, 1]$. The subdifferential of the indicator of $K$ (which is convex if and only if $K$ is convex) is given by $\partial \psi_{K}(x)=\{0\}$ if $x \in \operatorname{Int}(K)$, $\partial \psi_{K}(x)=N_{K}(x)$ if $x \in \partial K, \partial \psi_{K}(x)=\emptyset$ if $x \notin K$, where $\partial K$ is the boundary of $K$, and $N_{K}(x)=\left\{z \mid z^{T}(\zeta-x) \leq 0\right.$, $\forall \zeta \in K\}$ is the outwards normal cone to $K$ at $x \in K$. The inwards tangent cone $V(x)$ is the polar cone to $N_{K}(x)$ and is defined as $V(x)=\left\{z \mid \forall \zeta \in N_{K}(x), \zeta^{T} z \leq 0\right\}$. Both the normal and tangent cones are convex and are defined at points $x$ of the boundary $\partial K$ where the normal direction is not uniquely defined [see Fig. 4 in a simple case where the sets $x_{p}+N_{\Phi}\left(x_{p}\right)$ are depicted]. Polarity is a generalization of orthogonality, for convex cones. In mechanics with bilateral holonomic constraints, the admissible velocities and the contact reaction belong to orthogonal spaces. Roughly, this generalizes to unilateral constraints by replacing these orthogonal spaces by the tangent and normal cones, respectively [70]. Generalizations of these notions to nonconvex sets $K$ and functions exist [79], [23], allowing, e.g., to state that if $f(x)=|x|+1$ for $x<0, f(x)=x$ for $x \geq 0$, then $\partial f(0)=(-\infty, 1]$.

A super-potential (or pseudopotential) of dissipation [66], [79], [39] is a convex lsc proper mapping $\varphi(\cdot) \geq 0, \varphi(0)=0$, such that $F \in \partial \varphi(v)$, where $F$ and $v$ are a pair of dual variables (in mechanics, force, and velocities). The conjugate of the lsc convex function $f(\cdot): \mathbb{R}^{n} \rightarrow \mathbb{R}$ is defined as $f^{*}(y)=\sup _{x \in \mathbf{R}^{n}}\left[x^{T} y-f(x)\right]$; see (16) for an example. One has $v \in \partial \varphi^{*}(F)$. Bilateral constraints with Coulomb friction can be expressed this way, as well as many other physical laws [33]. Nonconvex superpotentials can be defined using Clarke's gradient [79]. There is also a strong apparatus of numerical algorithms associated to such analytical tools [2], [92], [74], [15].

The conventional notation $\partial$. for boundaries of sets, partial differentiation and subdifferential, is kept throughout the paper. The gradient $\nabla h(q)=\left(\partial h / \partial q_{1}, \ldots, \partial h / \partial q_{n}\right)^{T}$ if $q^{T}=$ $\left(q_{1}, \ldots, q_{n}\right)$. If $h(\cdot)$ is differentiable, then $\partial h(q)=\nabla h(q)$.

\section{EXAMPLES}

Some examples of systems that may be recast into the framework of (1) are presented. In view of the list provided in the introduction, the following examples are only a few cases of CS (see [87, Ch. 4] for other examples). We omit to indicate initial conditions. Let us notice that when solutions possess jumps, then the initial data in (1) have to be taken as a left-limit $x\left(\tau_{0}^{-}\right)=x_{0}$ to allow for initial jumps [71]. 
Example 2: (Mechanical system with frictionless unilateral constraint)

$$
\left\{\begin{array}{l}
M(q) \ddot{q}+F(q, \dot{q})=E u+\nabla h(q) \lambda \\
0 \leq y \perp \lambda \geq 0 \\
y-h(q)=0 \\
\text { Collision mapping. }
\end{array}\right.
$$

In (3) $x^{T}=\left(q^{T}, \dot{q}^{T}\right) \in \mathbb{R}^{n}$ and the other terms may be identified easily. The second line in (3) may be called the Hertz-Signorini-Moreau conditions [25]. According to the discussion in Section II-B, the associated automaton has $2^{m}$ states. When $h(q)=C q$, then one may define $y=q, \Lambda(\lambda)=-C^{T} \lambda$ and write the complementarity relation as $\mathcal{C}^{\star} \ni q \perp \Lambda(\lambda) \in \mathcal{C}$ with $\mathcal{C}^{\star}=\left\{q \in \mathbb{R}^{n / 2} \mid C q \geq 0\right\}$ and $\mathcal{C}=\left\{v \in \mathbb{R}^{n / 2} \mid v^{T} q \leq 0\right.$, $\left.\forall q \in \mathcal{C}^{\star}\right\}$. The dynamics in (3) encompasses manipulators performing complex robotic tasks (e.g., models for deburring tasks which have considerable importance in manufacturing industry [85] and many other machining tasks) as well as controlled Lagrangian CS. Important applications also exist in aerospace (pick up tasks with autonomous robots in zero-gravity fields, tethered satellites, landing aircrafts). The first use of (3) in Control and Robotics can be found in [45]. Models as in (4) including also Coulomb friction have been proved experimentally quite valuable for somewhat complex systems $(n / 2 \approx 10$, $m \approx 20$ ) in industrial applications for virtual prototyping of circuit breakers [1]. The existence and uniqueness of solutions $q \in \mathrm{AC}, \dot{q} \in \mathrm{RCLBV}$ is guaranteed if and only if all data are piecewise analytic [5].

If $h(q)>0$ then $\lambda=0$. If $h(q(t))=0$ on $\left[t^{\prime}, t^{\prime \prime}\right], t^{\prime \prime}>t^{\prime}$, then $(d h / d t)(q(t), \dot{q}(t))=0$ and $\left(d^{2} h / d t^{2}\right)(q(t), \dot{q}(t), \ddot{q}(t))=$ 0 on $\left(t^{\prime}, t^{\prime \prime}\right)$. Thus, $\lambda$ is calculated on $\left(t^{\prime}, t^{\prime \prime}\right)$ from the LCP $0 \leq \lambda \perp \ddot{h}(q(t), \dot{q}(t), \ddot{q}(t)) \geq 0$, which involves both the complementarity conditions and the continuous dynamics [similarly as for systems $\left(S_{1}\right)$ and $\left(S_{3}\right)$ in (2)]. The LCP $(\lambda)$ matrix is equal to $\nabla h(q)^{T} M^{-1}(q) \nabla h(q) \geq 0$ so the LCP has at least one solution [64]. If $\lambda\left(t^{\prime \prime}\right)=0$, detachment from the boundary $h(q)=0$ occurs if $\left(d^{2} h / d t^{2}\right)\left(q\left(t^{\prime \prime}\right), \dot{q}\left(t^{\prime \prime}\right), \ddot{q}\left(t^{\prime \prime}\right)\right)>0$. If $h(q(t))=0$ and $\nabla h(q(t))^{T} \dot{q}\left(t^{-}\right)<0$, then $\dot{q}(\cdot)$ jumps at $t$, which is an impact time $t_{k}$. At impact times $\lambda$ is a Dirac measure and the Lagrange dynamics becomes algebraic [12].

When $\Phi=\{x \mid h(q) \geq 0\}$ is convex, the dynamics in (3) can be equivalently rewritten as

$$
\left\{\begin{array}{cc}
-M(q) \ddot{q}-F(q, \dot{q})+E u \in \partial \psi_{\Phi}(q) & \text { when } t \neq t_{k} \\
\dot{q}\left(t_{k}^{+}\right)=\arg \min _{z \in V\left(q\left(t_{k}\right)\right)} \frac{1}{2} Z^{T} M\left(q\left(t_{k}\right)\right) Z, \quad k \geq 0
\end{array}\right.
$$

with $Z=z-\dot{q}\left(t_{k}^{-}\right)$, so that (4b) is Moreau's collision mapping [70]. In (4) $V\left(q\left(t_{k}\right)\right)$ is the convex tangent cone to $\Phi$ at $q\left(t_{k}\right)$. If $m=1$ this mapping reduces to Newton's law in (9), with $e=0$. A coefficient of restitution can be introduced in the mapping in (4b); see [71], [59], and [12]. It is crucial to recall that $t_{k}$ in (4) is a solution of a nonlinear equation depending on the state [12, Sec. 1.3.1] and is most often only implicitly defined. One may also replace the right-hand side of (4a) by the (more restrictive) inclusion $\in \partial \psi_{V(q(t))}\left(\dot{q}\left(t^{+}\right)\right) \subseteq \partial \psi_{\Phi}(q)$ [70], which incorporates the collision mapping in (4b). Actually the term $\psi_{\Phi}(q)$ can be interpreted as a displacement potential, whereas
$\psi_{V(q(t))}\left(\dot{q}\left(t^{+}\right)\right)$can be interpreted as a velocity potential [35]. Both are associated to the unilateral constraints $h(q) \geq 0$, and physically state the impenetrability of the bodies in contact. The notion of MDIs is introduced in Appendix A. From (4) one can obtain Moreau's second-order sweeping process [53], i.e., the MDI

$$
\begin{aligned}
-M(q(t)) \frac{d v}{d \mu}-F(q(t), v(t)) \frac{d t}{d \mu}+ & E u \frac{d t}{d \mu} \\
& \in \partial \psi_{V(q(t))}\left(\dot{q}\left(t^{+}\right)\right)
\end{aligned}
$$

where $q(t)-q(0)=\int_{0}^{t} v(z) d z$, and the measure $d \mu$ can be chosen as indicated in Section VI-B. This formalism is the proper extension of Lagrange equations to systems with unilateral constraints. It may be more useful than the complementarity one when dealing with existence of fixed points or stability considerations. The domain $\Phi$ may be described as in (3) without being convex. One may then use the notion of nonconvex superpotential or generalized gradient [79] to write (4a), see, e.g., [23, Prop. 2.4.12]. The right-hand side of the inclusion (5) encompasses both cases where $\dot{q}(\cdot)$ is continuous [in which case $\dot{q}\left(t^{+}\right)=\dot{q}(t)$ ] and discontinuous (in which case $t=t_{k}$ for some $k$ ). At $t=t_{k}$ then the MDI in (5) becomes the classical algebraic shock dynamics.

Remark 3: Nondifferentiable points are those configurations $q \in \partial \Phi$ where the normal direction to $\partial \Phi$ is not uniquely defined, however the normal cone exists. The formalism in (3) precludes so-called reintrant corners, or "peaks" [35], but does not imply convexity of $\Phi$. For instance, the domain in $\mathbb{R}^{2}$ defined as $q^{T}=(x, y), x^{2}+y^{2} \geq 1, y \geq 0$, is not convex, but locally around $(-1,0)$ and $(1,0)$ it is convex (in the terminology of [23, def. 2.4.6], the set $\Phi$ is regular at $(-1,0)$ and $(1,0)$ ).

Example 3 (Mass + Linear Spring-Damper): The dynamics of a mass with position $q$, that rebounds on a spring-damper system with position $\xi$, and where the contact force $\lambda \in \mathbb{R}$ has to remain nonnegative and satisfies a complementarity relation with the distance $y$, is given by

$$
\left\{\begin{array}{l}
\dot{x}=A x+B \lambda \\
0 \leq y=C x \perp \lambda \geq 0
\end{array}\right.
$$

where $x^{T}=(q, \dot{q}, \xi), k$ the stiffness and $c$ the damping coefficients

$$
\begin{aligned}
A & =\left(\begin{array}{ccc}
0 & 1 & 0 \\
0 & 0 & 0 \\
0 & 0 & -k / c
\end{array}\right) \quad B^{T}=\left(\begin{array}{lll}
0 & \frac{1}{m} & -\frac{1}{c}
\end{array}\right) \\
C & =\left(\begin{array}{ccc}
1 & 0 & -1
\end{array}\right) .
\end{aligned}
$$

The contact and noncontact phases correspond to $y=0(\Rightarrow \lambda=$ $-c \dot{q}-k q \geq 0)$ and $y>0(\Rightarrow \lambda=0)$, respectively. When $c=0$ the graph of the piecewise-linear relation between $\lambda$ and $q$ is depicted in Fig. 3(a). Notice that in this case $\lambda=\max (k q, 0)$, and "max" relations possess a complementarity formalism [41]. This example shows that unilaterality does not imply rigidity. However, it implies impenetrability.

If $C x(t)=0$ on $\left[t^{\prime}, t^{\prime \prime}\right]$, then the complementarity condition $0 \leq C \dot{x} \perp \lambda \geq 0$ holds on $\left(t^{\prime}, t^{\prime \prime}\right)$. Since $C B=1 / c>0, \lambda$ is at time the unique solution of the $L C P 0 \leq \lambda \perp C A x(t)+$ $C B \lambda \geq 0$. Transitions from noncontact to contact occur with a continuous state $x(\cdot)$. 


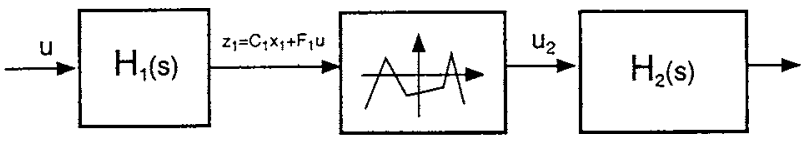

Fig. 1. Systems with a piecewise linear interconnection.

Fig. 1 illustrates the cascade connection of two linear systems with transfer functions $H_{1}(s)$ and $H_{2}(s)$, and with a piecewise linear interconnection between the first system's output $z_{1}$ and the second system's input $u_{2}$. Some typical piecewise linear characteristics are depicted in Fig. 3.

Example 4 (Dead-Zone, MOS Transistor): Let us consider a dynamical system as depicted in Fig. 1 with the piecewise linear characteristic in Fig. 3(b) with $b=a$. The dynamics is given by

$$
\left\{\begin{array}{l}
\dot{x}_{1}=A_{1} x_{1}+B_{1} u \\
\dot{x}_{2}=A_{2} x_{2}+a B_{2}\left(C_{1} x_{1}+F_{1} u+d\right)+E \lambda \\
y_{1}=\lambda_{1}-C_{1} x_{1}-F_{1} u-d \\
y_{2}=\lambda_{2}-C_{1} x_{1}-F_{1} u+d \\
0 \leq y \perp \lambda \geq 0
\end{array}\right.
$$

where $E=a B_{2}(-1,1) \cdot \operatorname{In}(7) x^{T}=\left(x_{1}^{T}, x_{2}^{T}\right)$, and $\operatorname{card}(L)=$ 3 . The three modes are $S_{1}=\left\{y_{1}>0\right.$ and $\left.y_{2}>0\right\}, S_{2}=\left\{y_{1}=\right.$ 0 and $\left.y_{2}>0\right\}, S_{3}=\left\{y_{1}=y_{2}=0\right\}$. When $0<b<+\infty$ and $a=+\infty$, Fig. 3(b) represents the characteristic of a MOS transistor (with $z_{1}$ a control voltage).

Example 5 (Saturation, Elasto-Plasticity): The dynamics of the CS in Fig. 1 where the piecewise-linear relation is as in Fig. 3(c) is given by

$$
\left\{\begin{array}{l}
\dot{x}_{1}=A_{1} x_{1}+B_{1} u \\
\dot{x}_{2}=A_{2} x_{2}+B_{2}\left(-1+\lambda_{1}-\lambda_{2}\right) \\
y_{1}=\lambda_{1}-C_{1} x_{1}-F_{1} u-1 \\
y_{2}=\lambda_{2}-C_{1} x_{1}-F_{1} u+1 \\
0 \leq y \perp \lambda \geq 0 .
\end{array}\right.
$$

Here, $\operatorname{card}(L)=3$ and the nonfeasible mode is $\left\{y_{1}>0\right.$ and $\left.y_{2}=0\right\}$. The three feasible modes are the same as in example 4.

In both examples 4 and 5 , the multiplier $\lambda$ is calculated at time $t$ as the unique solution of a LCP whose matrix is the identity, similarly as in $\left(S_{2}\right)$ in (2) with $\alpha=1$.

Example 6 (Painlevé System): Coulomb friction lends itself to a representation by complementarity relations, since the graph in Fig. 3(f) is monotone with $a \geq 0$ [so that $u_{2} \in \partial g\left(z_{1}\right)$ for some convex $g(\cdot)]$. Consequently the dynamics of a slender rod subject to a unilateral point-contact with Coulomb friction (coefficient $\mu>0$ ), as in Fig. 2, is given by

$$
\left\{\begin{array}{l}
m \ddot{q}_{1}=\lambda_{t} \\
m \ddot{q}_{2}=-m g+\lambda_{3} \\
I \ddot{\theta}=u+l\left[-\cos (\theta) \lambda_{3}+\sin (\theta) \lambda_{t}\right] \\
0 \leq y \perp \lambda \geq 0 \\
\quad \text { Newton's impact law: } \dot{q}^{T}\left(t_{k}^{+}\right) \nabla h\left(q\left(t_{k}\right)\right) \\
\quad=-e \dot{q}^{T}\left(t_{k}^{-}\right) \nabla h\left(q\left(t_{k}\right)\right) \\
F=\arg \max _{z \in \mathcal{C}(q)}\left[-z^{T} \dot{q}\right]
\end{array}\right.
$$

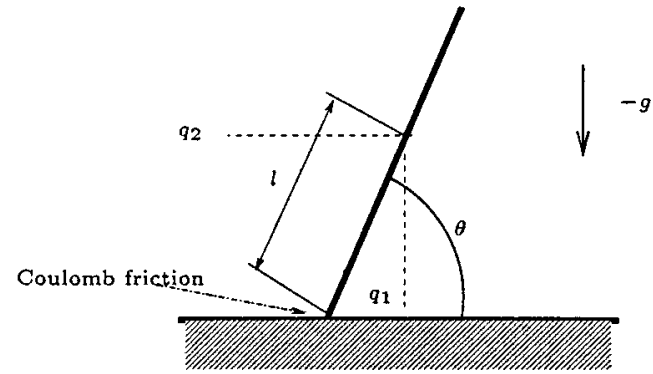

Fig. 2. Painlevé system.

where $\lambda^{T}=\left(\lambda_{1}, \lambda_{2}, \lambda_{3}\right), \lambda_{t}=\left(\mu \lambda_{3} / 2\right)\left(\lambda_{2}-\lambda_{1}\right), \lambda_{1}+\lambda_{2}=$ $2, y_{1}-y_{2}=\dot{q}_{1}+l \dot{\theta} \sin (\theta), h(q)=y_{3}=q_{2}-l \sin (\theta)$, and $\left(q_{1}, q_{2}\right)$ are the center of mass coordinates and $\theta$ is the rod orientation, $q^{T}=\left(q_{1}, q_{2}, \theta\right), e \in[0,1]$. The vector $F \in \mathbb{R}^{3}$ is the generalized contact reaction and $\mathcal{C}(q)$ is the friction cone in generalized coordinates [12, p. 328]. In (9), there may exist velocity jumps during sliding regimes $\left\{y_{1}=0\right.$ and $\left.y_{2}>0\right\}$ and $\lambda_{3}$ may diverge to infinity in the vicinity of some critical points (the state remaining however continuous at such critical points). These two phenomena are a consequence of the coupling between complementarity conditions and Coulomb friction, which yields a very particular type of singular differential equation and may destroy the convexity of the underlying quadratic program for calculating $\lambda$ (see Appendix B) [12], [34]. One notes that $f(x, u, \lambda)$ in (9) is nonlinear in $\lambda$. Though solutions of (9) are such that $q \in \mathrm{AC}$ and $\dot{q} \in \mathrm{RCLBV}$ [95], uniqueness fails [34], and a complex behavior similar to that of example $\left(S_{2}\right)$ with $\alpha=-1$ in (2), can occur [55].

The multiplier $\lambda$ in (9) does exist as a measure. Compared to (3) the coupling between Coulomb friction and unilaterality complicates the definition of (9) as a hybrid dynamical system since it creates new modes. As a consequence state (velocity) reinitialization may occur at impacts but also in the slidingmotion mode.

Example 7 (Square Relation): Let us consider now the system in Fig. 3 with the set-valued relation whose graph is the square characteristic in Fig. 3(d)

$$
\left\{\begin{array}{l}
\dot{x}_{1}=A_{1} x_{1}+B_{1} u \\
\dot{x}_{2}=A_{2} x_{2}+B_{2}\left(-1+2 \lambda_{1}-2 \lambda_{2}-2 \lambda_{3}+2 \lambda_{4}\right) \\
0 \leq y \perp \lambda \geq 0 \\
\lambda_{2}-y_{2}=\lambda_{1}-y_{1}-1 \\
\lambda_{3}-y_{3}=\lambda_{1}-y_{1}-2 \\
\lambda_{4}-y_{4}=\lambda_{1}-y_{1}-3 \\
\lambda_{5}-y_{5}=\lambda_{1}-y_{1}-4 \\
1-2 \lambda_{2}+2 \lambda_{3}+2 \lambda_{4}-2 \lambda_{5}=C_{1} x_{1}+F_{1} u .
\end{array}\right.
$$

Here, $\operatorname{card}(L)=4: S_{1}=\left\{C_{1} x_{1}+F_{1} u=1\right.$ and $-1<$ $\left.u_{2} \leq 1\right\}=\left\{y_{1}=0, y_{2} \in[0,1), y_{3} \in[1,2), y_{4} \in[2,3)\right.$, $\left.y_{5} \in[3,4)\right\}, S_{2}=\left\{u_{2}=1\right.$ and $-1<C_{1} x_{1}+F_{1} u \leq$ $1\}=\left\{y_{1}=y_{2}=0, y_{3}=1, y_{4}=2, y_{5} \in[3,4)\right\}$ and so on for $S_{3}$ and $S_{4}$. Notice that the characteristic does not span the whole $x$-axis, i.e., $\mathrm{PL}_{\text {square: }}[-1,1] \rightarrow[-1,1]$. This is quite 

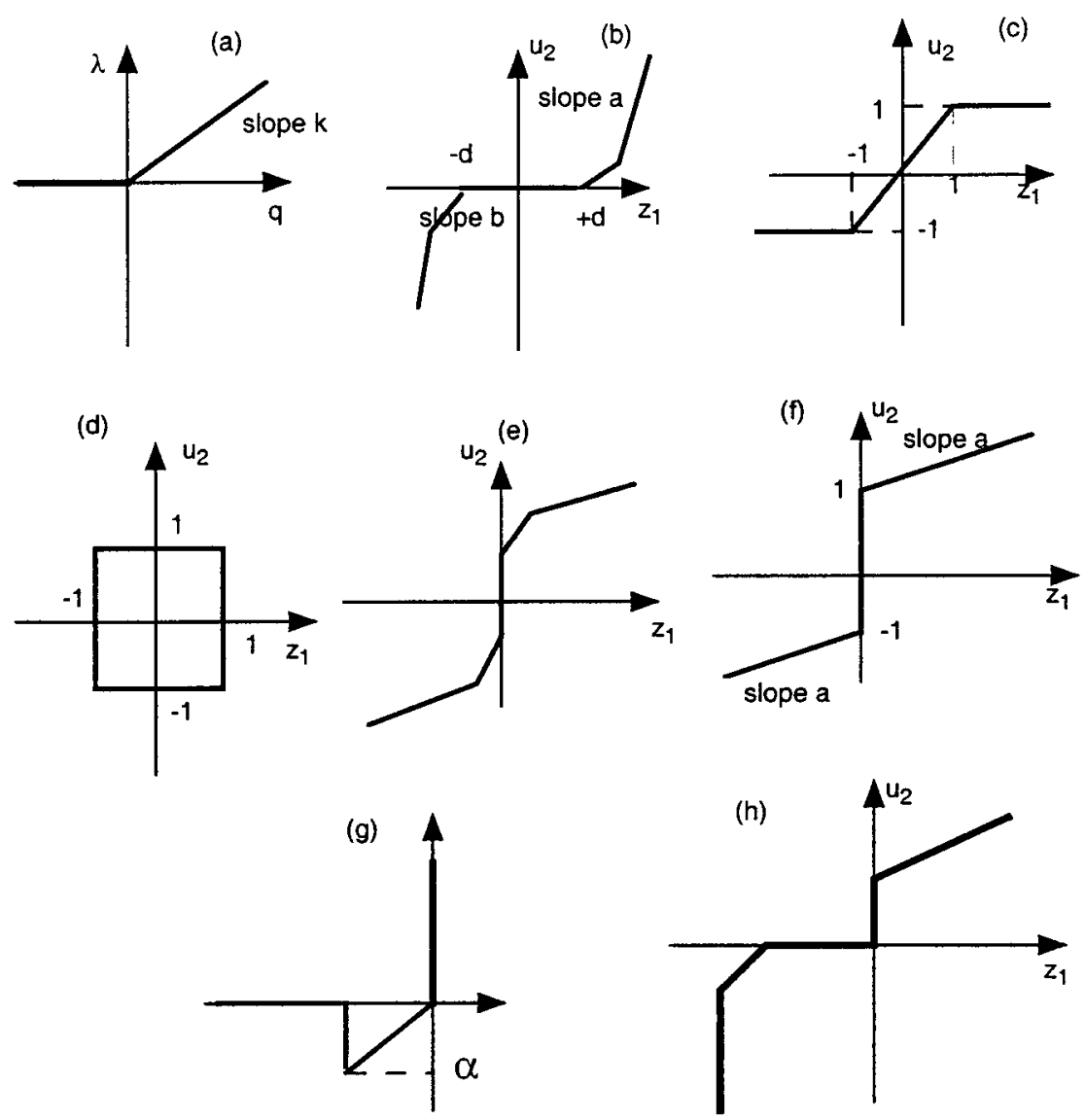

Fig. 3. Graphs of the piecewise-linear relations.

similar to the corner law characteristic $\mathrm{PL}_{\text {corner }}: \mathbb{R}^{+} \rightarrow \mathbb{R}^{+}$, as in Fig. 3(g) with $\alpha=0$ (the case $\alpha<0$ represents adhesive contact which may be encountered with rubber support, or in micro-robotics where van der Waals forces play a role in the contact). Let us say that such graphs contain some unilateral effects. Clearly similar developments can be made for the graphs in Fig. 3(e) (fluid with two viscosity ranges [79]) and Fig. 3(f) (rigid viscoplastic material characteristic; the case $a<0$ represents relaxation effects and the graph is non longer monotone, indicating a loss of dissipativity with consequences on stability); Fig. 3(h) (unilateral and adhesive effects [30] where $z_{1}$ is a displacement and $u_{2}$ is a contact impulse).

Example 8 (Electrical Circuit With Ideal Diode): A simple electrical circuit containing an ideal diode whose characteristic is a corner law as in Fig. 3(g) with $\alpha=0$, a current source and an inductor mounted in parallel, possesses the following dynamics:

$$
\left\{\begin{array}{l}
\dot{x}=\lambda \\
y=x+u \\
z=-\lambda \\
0 \leq y \perp \lambda \geq 0 \\
\text { State reinitialization rule }
\end{array}\right.
$$

where $x(t) \in \mathbb{R}$ is the inductor current, $\lambda(t) \in \mathbb{R}$ is the voltage across the diode, $-y(t)$ is the current across the diode, $u(t)$ is the current variable of the current source. Interestingly enough the dynamics of a yo-yo [56] and (11) are also quite similar, since the input $u(t)$ acts directly in the constraint $h(\cdot)$.

Example 9 (Generalized Nonholonomic Constraints): The dynamics of a particle in $\mathbb{R}^{3}$ with coordinates $\left(q_{1}, q_{2}, q_{3}\right)$, subject to the nonholonomic constraint

$$
A(q)^{T} \dot{q}=\left(q_{2}^{2}-q_{1}^{2}-q_{3}\right) \dot{q}_{1}+\left(q_{3}-q_{2}^{2}-q_{1} q_{2}\right) \dot{q}_{2}+q_{1} \dot{q_{3}}=0
$$

is studied in [24]. It is shown that the loss of rank of $A(q)$ when the trajectories enter some subset, implies the need for a state reinitialization to integrate the motion. Also the multiplier $\lambda$ associated to the constraint may diverge to infinity when the trajectories approach the singular set $\left\{q_{1}=0, q_{3}=q_{2}^{2}\right\}$. This is therefore an example of a system with a bilateral constraint (however, it can be easily recast in the framework of unilaterally constrained systems by expressing the equality in (12) as two inequalities), that yields a behavior conformable to that of the Painlevé system in example 6.

OP 1: In view of examples 6 and 9, extend the studies on singular differential equations to link Painlevé-like problems, dynamical systems with generalized constraints, and singular systems $E(x) \dot{x}=f(x)$, where $\operatorname{rank}(E(x))$ is not constant [84].

Other examples of dynamical systems that can be cast in a complementarity framework can be found in [8], [87], [41], 
[40], and [99]. From examples 3-5, 7, and 8, it follows that an important subclass of systems as in (1) is given by

$$
\left\{\begin{array}{l}
\dot{x}=A x+B u+E \lambda+\bar{e} \\
G y+C x+F u+D \lambda+\bar{d}=0 \\
0 \leq y \perp \lambda \geq 0 \\
\text { State } x(\cdot) \text { reinitialization rule. }
\end{array}\right.
$$

Systems as in (13) may be named ACS. It may be assumed that $G \neq 0$ to avoid meaningless complementarity conditions. However, as example 7 shows, $G$ is not necessarily full rank.

Remark 4: Some of the presented examples have already been thoroughly studied in the control literature without resorting to any complementarity framework (e.g., example 3 with $c=0$, or examples 4 (dead-zone), 5 with PL at the input [98]). However, on the one hand this is not true for all CS (by far). On the other hand, it is expected that the existing works, when replaced in a more general context, may provide some useful insights for the analysis of other systems. The fact that all these systems share a common structure cannot be passed over.

State reinitializations: The examples in (2) have shown that some CS can be integrated without state jump, whereas others have discontinuous solutions. As alluded to in example 6, the fact that a discontinuous state reinitialization is needed or not, may not always be evident. Let us consider (1) disregarding state reinitialization (consequently excluding initial state jumps), and such that assumption 1 holds. By construction the system is initialized in a certain mode $S_{i}$. The central question is: if the conditions for transition into another mode are met, can one integrate the system without reinitializing $x(\cdot)$ ? In other words, is continuation in another mode possible with a continuous $x(\cdot)$ or not? There are two main steps in this problem: determine the conditions such that state jumps are necessary, then define a rule for state reinitialization. As the mechanical systems (3) and (6) show, the relative degree $r_{y \lambda}$ between $y$ and $\lambda$ plays a major role in the need for state jump. In (3) $r_{y \lambda}=2$, so at $t_{k}$ no bounded $\lambda$ exist to solve $0 \leq \dot{y} \perp \lambda \geq 0$ if $\dot{y}\left(t_{k}^{-}\right)<0$. In (6), $r_{y \lambda}=1$ and the LCP $0 \leq \dot{y} \perp \lambda \geq 0$ always has a unique bounded solution, which allows the system to satisfy $y \geq 0$ without state jump. Consider now (7). Then one can always compute a unique $\lambda$ as a solution of a LCP $0 \leq y \perp \lambda \geq 0$, since the LCP matrix is the identity matrix so that $r_{y \lambda}=0$. Thus, no state reinitialization is needed to respect the condition $y \geq 0$ : a bounded $\lambda\left(x_{1}, u\right) \geq 0$ suffices. This can be generalized to (13), where the matrix $-G^{-1} D$ should be a $P$-matrix.

A geometric approach has been presented in [26] for systems $\dot{x}=f(x)+g(x) u, y=h(x), \mathcal{C}^{\star}=\mathbb{R}^{+}$which brings an answer to the first step. The theory is based on the use of relative degrees between $y$ and $u$ to characterize the set $h(x)=0$ and builds up possible reinitialization mappings. General state reinitialization rules are proposed in [42], [20], [21], and [24], for ACS as in (13), with $\bar{e}=0, G=-I_{m}, \bar{d}=0, F=0, B=0$. They extend Moreau's rule in (4). They can be formulated as quadratic programs or Cone $\mathrm{CP}$, which is of great usefulness for numerical simulations. The principle of maximal dissipation (see (9), sixth line) that is used to avoid penetration of the state into inconsistent zones for the Painlevé system in example 6, is another example of state reinitialization rule.

The choice of a suitable reinitialization map is a crucial step. Simple examples show that one has to choose it in accordance with the continuous dynamics (consider e.g., two masses which collide, moving on a straight line: linear momentum conservation precludes the use of some "imaginary" impact law such that both masses stop after any shock).

\section{RELATIONSHIPS BETWEEN CS AND OTHER HYBRID MODELS}

Differential Inclusions: It has already been pointed out in example 2 that there is a strong link between CS and some kind of DI. The dynamics in (13) can be equivalently rewritten as a differential-algebraic inclusion

$$
\left\{\begin{array}{l}
-\dot{x}+A x+B u+\bar{e} \in E \partial \psi_{\left(\mathbf{R}^{+}\right)^{m}}(y) \\
G y+C x+F u+\bar{d} \in-D \partial \psi_{\left(\mathbf{R}^{+}\right)^{m}}(y) \\
\text { State } x(\cdot) \text { reinitialization rule. }
\end{array}\right.
$$

The DI $\dot{x} \in F(x)$ almost everywhere, with $F(\cdot)$ upper-semicontinuous, $F(x) \neq \emptyset$ is convex and compact for all $x$, and $F(\cdot)$ satisfies linear growth conditions [i.e., $z \in F(x) \Rightarrow\|z\| \leq$ $a\|x\|+b$ for some $a>0$ and $b>0$ ], is widely studied, see [28]. Then AC solutions exist. In general, such DI and CS as in (1) are quite different. A case when they drastically differ is unilaterally constrained mechanical systems, since the linear growth and compactness conditions fail. Another example is in (11), which can equivalently be rewritten as $-\dot{x} \in \partial \psi_{[-u(t),+\infty)}(x)$. Therefore, it can be recast into Moreau's first-order sweeping process [68], [63], [53], i.e., DI of the form $-\dot{x} \in \partial \psi_{C(t)}(x)$ where $C(t)$ is a convex set depending on time.

However, for certain CSs, they coincide. This is the case of the following one degree-of-freedom mechanical system with Coulomb friction (corresponding to the graph in Fig. 3(f) with $a=0$ )

$$
\left\{\begin{array}{l}
m \ddot{q}=u+\frac{\mu m g}{2}\left(\lambda_{2}-\lambda_{1}\right) \\
y_{1}-y_{2}=-\dot{q} \quad \lambda_{1}+\lambda_{2}=2 \\
0 \leq y \perp \lambda \geq 0
\end{array}\right.
$$

where $\lambda^{T}=\left(\lambda_{1}, \lambda_{2}\right), y^{T}=\left(y_{1}, y_{2}\right)$ and the analogy with (13) or (14) can be made. The dynamics in (15) can be equivalently written as [71]

$$
-m \ddot{q}+u \in \mu m g \partial \psi_{[-1,1]}^{\star}(\dot{q})
$$

where $\psi_{[-1,1]}^{\star}(\cdot)=|\cdot|$ is the conjugate function of the indicator $\psi_{[-1,1]}(\cdot)$ and $\partial|\cdot|$ satisfies the aforementioned standard hypotheses on $F(\cdot)$. It is a convex superpotential. Notice that (16) can also be cast into the framework of Filippov's systems, i.e., a special sort of DI. The Zhuravlev-Ivanov transformation can be used to transform mechanical systems as in (3) and with $m=1$ into Filippov's inclusions [12]. 
OP 2: Determine the conditions on the matrices in (13) such that indeed (14) is a DI: $\dot{x} \in F(x, u)$ with $F(x, u)$ satisfying some standard hypotheses [28, Secs. 5 and 6], with an AC solution. Uniqueness is more complex to establish but is also an important and largely open issue [83].

Due to the numerous studies on the sweeping process [68], [63], [53], the following is of interest.

OP 3: Determine the conditions such that (14) is a first-order sweeping process, and derive well-posedness results for such ACS (in particular the nature of solutions depending on the set of admissible controllers $u$ ).

OP 3 may be a preliminary step into some cross-fertilization between mathematical studies on various types of DI ([10], [53], [63], [50], [28], and the references therein), and complementarity approaches [20], [21], [40]-[43], [89], [88].

Measure Differential Equations (MDE): Specific notions of solutions have been introduced for MDE of the form $\dot{x}=f(x, t)+g(x, t) \dot{u}$ with $u \in$ RCBLV [78], or even with quadratic terms in $\dot{u}$ [9]. Other types of MDEs are considered in [4] with piecewise continuous solutions, of the form $\dot{x}=f(x, t)$ if $t \neq t_{k}(x), x\left(t_{k}^{+}\right)=g\left(x\left(t_{k}^{-}\right)\right)$if $t=t_{k}(x)$ for some sequence $\left\{t_{k}(x)\right\}_{k \geq 0}$. It is clear that such models and (1) are of different nature. Significant differences between MDE and CS are stated in [12, Sec. 1.4.2, Ch. 7], and in [15]. Actually, the complementarity conditions in (1) play a major role in the dynamics and in all the properties of such systems. This does not prevent some analytical tools (like stability notions) to apply both to such MDE and certain CS, as pointed out in [12, Remark 8.12]. But this is not true in general [87, Sec. 1.2.5].

Piecewise Smooth Systems (PSS): PSS may be defined as $\dot{x}=f_{i}(x, t)$ if $x \in \mathcal{D}_{i}, \cup_{i} \mathcal{D}_{i}=\mathbb{R}^{n}$, each $\mathcal{D}_{i}$ has a nonempty interior, $\mathcal{D}_{i} \cap \mathcal{D}_{j}$ is either a zero-measure set included in $\partial \mathcal{D}_{j}$ or in $\partial \mathcal{D}_{i}$ (e.g., $x \geq 0$ and $x \leq 0$ ), or $\emptyset$ (e.g., $x \leq 0$ and $x>0$ ). The $f_{i}(\cdot, \cdot)$ are smooth vector fields, $i$ is an index (positive integer). Mimicking OP 2 and OP 3, let us state the following.

OP 4: What CS can be represented equivalently as PSS, and vice versa?

Consider examples 3 (with $c=0$ ), and 4 (with $a<+\infty$ ) and 5 with no input (i.e., $u \equiv 0$ ). They are PSS [such is not the case for (3)]. Certainly the definition of the vector fields on the intersections $\mathcal{D}_{i} \cap \mathcal{D}_{j}$ plays a role, since it is related to graphs (filling the gaps at discontinuity points) and maximality for monotone operators. This is clearly illustrated by the various definitions one may give to the signum function. $\mathbf{O P} \mathbf{4}$ has been clarified in [43] when the continuous dynamics is in a discrete-time representation. Related results are also in [22]. It follows from a result in [99] that all systems with a piecewise linear connection (see Fig. 1) can be represented as an ACS in (13).

OP 5: Study conditions on the Cone CP: $\mathcal{C}^{\star} \in y \perp \Lambda(\lambda) \in \mathcal{C}$ and on the function $g(y, \lambda, u, x, t)$, for (1) to be PSS. Discover state variable changes (possibly nonsmooth, noninvertible) such that the transformed trajectories are time-continuous. How may one use conjugacy of convex functions in this setting?

More generally, there is certainly a strong need for clarifying the links between numerous models of hybrid and nonsmooth systems. This section only aims at pointing out possible paths for few of them.
For instance, let us notice in passing the following equivalences:

$$
\begin{aligned}
\left(S_{1}\right) & \Leftrightarrow \dot{x}+x+a \in-\partial \psi_{\mathbf{R}^{+}}(x) \\
& \Leftrightarrow\left\{\begin{array}{l}
\langle\dot{x}+x+a, v-x\rangle \geq 0, \forall v \in \mathbb{R}^{+} \\
x(\cdot) \geq 0
\end{array}\right. \\
& \Leftrightarrow \dot{x}=-P_{\mathbb{R}^{+}}(x+a)
\end{aligned}
$$

with $\alpha=1$ in $\left(S_{1}\right)$ in (2) and $P_{\mathbf{R}^{+}}$is a linear projection. The last-but-one equivalence is between a differential inclusion and a linear evolution variational inequality [37]. The last formalism is called a projected dynamical system (PDS) [75] and the equivalence uses basic convex analysis [44, Prop. A5.3.3]. VIs are another type of dynamical system which is widely used in some branches of applied mathematics [37], [75]. As (17) shows, in some cases DI, VI, CS, PSS, and PDS are only different formalisms for the same system.

\section{Controllability Issues}

The system (1) is controllable on $\mathbb{R}^{n}$ if for any pair $\left(x_{0}, x_{1}\right)$ of states, there is an admissible input $u:\left[\tau_{0}, T\right] \rightarrow \mathbb{R}^{p}$ such that $x\left(T^{+}\right)=\varphi\left(T^{+} ; \tau_{0}, x_{0}, u\right)=x_{1}$. There is a unique notion of controllability for linear time-invariant systems. However, such is not the case for nonlinear systems. Many relaxed notions of controllability exist in this case, which are not in general equivalent. Roughly, the controllability properties are characterized by the reachable sets [77]

$$
\begin{aligned}
R^{V}\left(x_{0}, T\right)= & \left\{x \in \mathbb{R}^{n} \mid \exists u:\left[\tau_{0}, T\right] \rightarrow \mathcal{U}\right. \text { such that the } \\
& \text { evolution of }(1) \text { satisfies } x\left(t^{+}\right) \\
= & \varphi\left(t^{+} ; \tau_{0}, x_{0}, u\right) \in V, \text { for all } \\
& \left.\quad t \in\left[\tau_{0}, T\right], \text { and } x\left(T^{+}\right)=x\right\}
\end{aligned}
$$

where $V$ is a neighborhood of $x_{0}$. It is assumed in this definition that assumption 1 holds at least on $\left[\tau_{0}, T\right]$. The system is accessible from $x_{0}$ when $R^{V}\left(x_{0}\right)=\cup_{\tau_{0} \leq t \leq T} R^{V}\left(x_{0}, t\right)$ contains a nonempty open set for any $V$ and sufficiently small $T>0$. A Lagrangian system is said to be equilibrium controllable [54] if for any two equilibrium points $q_{0}$ and $q_{1}$, there exists an input $u(t)$, and a solution $q(t), t \in\left[\tau_{0}, T\right]$, such that $q\left(\tau_{0}\right)=q_{0}$, $q(T)=q_{1}, q(t) \in V$ for all $t \in\left[\tau_{0}, T\right]$, and $\dot{q}\left(\tau_{0}\right)=\dot{q}(T)=0$.

\section{A. Example}

Let us illustrate briefly on an example how the complementarity relations and the state reinitialization rule, may influence the controllability properties of a dynamical system. To this end, let us first consider the controllable dynamics $\left\{\begin{array}{l}\dot{x}_{1}=x_{2} \\ \dot{x}_{2}=u\end{array}\right.$. Let us add a unilateral constraint $x_{1} \geq 0$ and an impact law $x_{2}\left(t_{k}^{+}\right)=-e x_{2}\left(t_{k}^{-}\right)$, with $e=0$. As a consequence all states $\left(x_{1}, x_{2}\right)=(0, b)$ with $b>0$ cannot be reached from any admissible state $x_{1} \geq 0$, using bounded inputs. If $x_{2}$ is RCLBV, then the states $(0, b)$ with $b<0$ cannot be reached neither, so that only the origin $(0,0)$ is reachable on $\partial \Phi$. However, the CS $\left(\lambda \geq 0, \lambda x_{1}=0, x_{1} \geq 0\right.$ and $\left.\dot{x}_{2}=u+\lambda\right)$ is equilibrium controllable. Let us now modify the controlled dynamics by adding $x_{2} \geq \varepsilon, \varepsilon>0$. Then, $\dot{x}_{1}>0$ in $\operatorname{Int}(\Phi), \Phi=\left\{x \mid x_{2} \geq \varepsilon\right\}$. Consequently the system may be rendered controllable only if 
negative jumps in $x_{1}$ are introduced on $\partial \Phi$, which may not be a good idea. Otherwise, the system is accessible only. As we have seen in example $\left(S_{1}\right)$ in (2), the external action $a$ can be used to steer $x(\cdot)$ inside $\Phi$.

\section{B. Admissible States and Inputs}

Let us first propose the following.

Definition 1: Let $u(\cdot)$ be a control input such that assumption 1 is satisfied. Then, $x(\cdot)=\varphi\left(\cdot ; \tau_{0}, x_{0}, u\right)$ is admissible for (1) at time $t \in\left(\tau_{0}, \tau_{0}+\varepsilon\right)$ if $x\left(t^{+}\right) \in \Phi=\{v \mid \exists \lambda$ such that $g(y, \lambda, u(t), v, t)=0$ and $\left.\mathcal{C}^{\star} \ni y \perp \Lambda(\lambda) \in \mathcal{C}\right\}$. The set $\Phi$ is the admissible set.

Therefore, clearly appears that one interpretation of the third and fourth lines in (1) is that they form a generalized constraint. Due to assumption 1, the right-limit $x\left(t^{+}\right)$always exists. From definition 1 admissibility applies to the right limit. One notices that admissibility does not preclude accumulations of jumps in $x(t)$. At times $t_{k}$ admissibility may be stated as: $x\left(t_{k}^{-}\right)$belongs to the domain of the state jump mapping. The notion of admissibility is a static notion to be checked at a time instant. For instance, the left limit $x\left(\tau_{0}^{-}\right)=x_{0}$ may be nonadmissible, so that the solution has to instantaneously jump to an admissible right limit. In $\left(S_{1}\right)$ in (2) with $\alpha=1$, only $x\left(\tau_{0}\right) \geq 0$ is admissible. In $\left(S_{2}\right)$ with $\alpha=1$, all $x\left(\tau_{0}\right)$ are admissible. In (3), $q\left(\tau_{0}^{-}\right)\left(=q\left(\tau_{0}^{+}\right)\right)$has to be admissible since $q(t)$ is AC. However one may need to consider higher derivatives of the signals to verify the admissibility. This is the case of example 2 in which one needs to differentiate $y$ twice to enable the calculation of $\lambda$ during phases where $y \equiv 0$, by constructing a suitable LCP. Consider example 5. Then $\lambda$ can be calculated as the solution of the LCP: $0 \leq y \perp \lambda \geq 0$, which always possesses a unique solution $\lambda\left(x_{1}, u\right)$ since the LCP matrix is positive definite (it is the identity matrix). In both cases, one sees that $\Phi$ does not depend on $u$. Let us consider now example 6 with $u=0$. The mathematical analysis [95], [34] shows that $\lambda_{1}$ and $\lambda_{2}$ can be Dirac measures so that $\dot{q}$ may jump, during sliding modes $\left\{y_{1}=0\right.$ and $\left.y_{2}>0\right\}$. Such discontinuities are a consequence of the coupling between the complementarity relations $0 \leq \lambda_{3} \perp y_{3} \geq 0$ and Coulomb friction. They are not due to any impact between the rod's tip and the ground, in which case $\lambda_{3}$ would be a Dirac measure. They prevent the system from violating the constraint $y_{3} \geq 0$ and from penetrating into an inconsistent subspace of the state space. Such inconsistencies are not rare, and practical examples show that they do occur for reasonable values of physical parameters [15], [12]. When $u \neq 0$ and differentiating twice $y_{3}$, one obtains that the contact force $\lambda_{3}$ is the solution of a LCP of the form $0 \leq A(\theta, \dot{\theta}, u)+B(\theta, \mu) \lambda_{3} \perp \lambda_{3} \geq 0$. Consequently the domain of inconsistent $(\theta, \dot{\theta})$ (see [12] or [34, Fig. 2]) depends on $u$. Moreover, if $u$ depends on $\lambda_{3}$, then the LCP matrix $B(\theta, \mu)$ is modified also. This may have important consequences on the controllability of the system, since the states which are a priori reachable must belong to $\Phi$. In the latter case, one might even imagine a bounded $u$ such that $\Phi=\emptyset$.

OP 6: Determine conditions on $f(x, u, \lambda), g(y, u, x, \lambda), \mathcal{C}$ such that $\Phi$ does not depend on $u$. Start with ACS as in (13). Classify CS for which $u(x, t)$ does not modify the properties of the CP to calculate $\lambda$, systems for which $u(\lambda)$ is forbidden, systems for which $u(x, t)$ may modify the shape of $\Phi$.

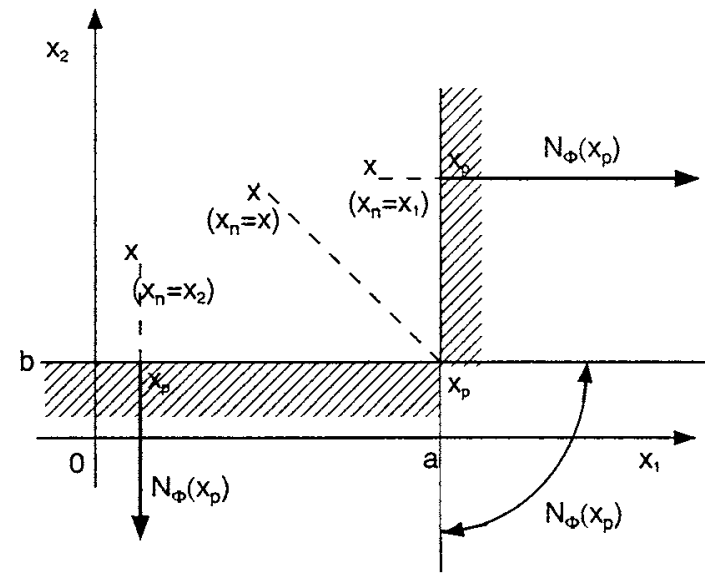

Fig. 4. Controllability in the normal cone.

OP 7: Determine the set $\mathcal{U}$ of admissible inputs such that $\Phi \neq \emptyset$, or such that $\Phi \supset R$, where $R$ is a given subset of the state space, for all $u \in \mathcal{U}$.

The notion of admissibility does not at all replace nor imply the uniqueness of solutions. Actually, a state $x_{0}$ may be admissible, and $\lambda$ be unique while nonuniqueness of solutions emanating from $x_{0}$ holds, or $\lambda$ may not be unique while solutions are, see [12, Sec. 5.4]. However. it is a preliminary notion which may help the control designer in planning which states are $a$ priori reachable or not. It is thus advocated that the accurate characterization of $\Phi$ is an important step in a control study. One should always speak of controllability over $\Phi$ (independent of $u$ ) for constrained systems, since $R^{V}\left(x_{0}, T\right) \subseteq \Phi$ for any admissible $x_{0}$. Admissible sets have been characterized in [20] for passive LCS and in [22] for a special class of piecewise linear systems.

Remark 5: The notion of viability of the input $u$ [16] also has to be considered. Let us consider $y=h(x)$ and $\mathcal{C}^{\star}=\mathbb{R}^{+}$. Roughly a control input is said to be viable on $\left[\tau_{0}, \tau_{0}+T\right]$, $T \geq 0$, if the trajectory $x(t)=\varphi\left(t ; \tau_{0}, x_{0}, u\right) \in \operatorname{Int}(\Phi)$. Consider again $\dot{x}_{1}=x_{2}, \dot{x}_{2}=u$, with $x_{1} \leq a, x_{2} \geq b, a>0$, $b>0$, see Fig. 4. If $x_{1}(0)=0$ then at $t \leq a / b$ the orbit hits the boundary $x_{1}=a$ whatever $u$. Thus, no input is viable for $T>a / b$.

Viability of the input may be crucial in some control studies [60]. Let us introduce the following notion.

Definition 2: Let $\Phi=\{x \mid h(x) \geq 0\}$ for (1), with differentiable components $h_{i}(\cdot), 1 \leq i \leq m$. Let us denote the projection of a solution $x(t)=\varphi\left(t ; t_{0}, x_{0}, u\right)$ on the boundary $\partial \Phi$ as $x_{p}(t)=\varphi_{p}\left(t ; t_{0}, x_{0}, u\right)$. Let us also denote the projection of the trajectories on $-N_{\Phi}\left(x_{p}\right)$ as $x_{n}(t)=\varphi_{n}\left(t ; t_{0}, x_{0}, u\right)$. Then, if the mapping $\left\{\begin{array}{c}\mathcal{U} \rightarrow-N_{\Phi}\left(x_{p}\right) \\ u \rightarrow \varphi_{n}\left(t ; \tau_{0}, x_{0}, u\right)\end{array}\right.$ is surjective for any $x_{p} \in \partial \Phi$, the system is said to be normal controllable (NC).

In other words, $\mathrm{NC}$ means that trajectories can be controlled in the normal direction to $\partial \Phi$, see Fig. 4 . The NC property implies the existence of a viable input, and the work in [26] can be used to characterize it. This notion is quite consistent with viability of sets in DI theory and is natural since the orbits evolve in some admissible sets. The novelty is that the set of forbidden states may not be constant. 


\section{Complementarity Mechanical Systems}

From the previous developments, it follows that solutions of CS possess the generic form (we drop all arguments for clarity of writing)

$$
\varphi=\varphi_{S_{j}} \circ \mathcal{F}_{j} \circ \underbrace{\varphi_{S_{j-1}}^{k} \circ \mathcal{F}_{j-1}^{k} \circ \varphi_{S_{j-1}}^{k-1} \circ \cdots \circ \varphi_{S_{j-1}}^{1} \circ \varphi_{S_{1}}}_{\text {possible finite accumulation }}
$$

where the DES path is $\mathcal{S}=\left(S_{j}, S_{j-1}^{k}, \ldots, S_{j-1}^{1}, \ldots, S_{1}\right)$ and each transition $S_{j-1} \rightarrow S_{j}$ is a priori done through a state $x(\cdot)$ reinitialization mapping $\mathcal{F}_{j}$. The solution between two switches in a mode $S_{j}$ is denoted as $\varphi_{S_{j}}=\varphi\left(t ; t_{j-1}^{+}, x\left(t_{j-1, j}^{+}\right), u\right)$, where $t_{j-1, j}$ is the transition $S_{j-1} \rightarrow S_{j}$ instant, and $t_{j-1, j} \leq$ $t \leq t_{j, j+1}$. In case of a finite accumulation of transitions, one has $k=+\infty$ in (19). Notice that there may be several infinities of events, even in simple systems (think of a bouncing ball). From (19) it follows that the controllability of (1) depends on $\mathcal{S}$ (what sequence?), the mappings $\mathcal{F}_{j}, 1 \leq j$ (do they help, bother, or are not concerned? Does bijectivity of these mappings allow one to ignore their effect on controllability?), the controllability properties in each mode $S_{j}$ in $\mathcal{S}$ [recall the $\varphi_{S_{j}}, 1 \leq j$, in (19) depend on $u$ ], and combination of all these ingredients. Some peculiarities of controllability of systems in (1) have been highlighted in the foregoing section. Let us consider (3). Then preimpact states with $h(q)=0$ and $\nabla h^{T}(q) \dot{q}<0$, are not reachable since $\dot{q}(\cdot)$ is right-continuous. More generally the work in [26] can be used to characterize reachable subsets of $\partial \Phi$.

The work in [19] considers the controllability properties of a mechanical system in each mode and combines them in order to check the equilibrium controllability. However it is assumed in [19] that at any state $x(t)$, one can switch to any mode $S_{i}$ instantaneously, i.e., there is a nonempty set of discrete controls for the event times $t_{i, j}$. Consequently, the derived criterion applies to systems with clamping devices (bilateral constraints that can be activated or deactivated at will), rather than to mechanical systems as in (3), see Remark 2. The work in [38] is also based on the controllability properties of a mechanical system in each mode. It applies to quasistatic systems (no drift, no collisions, inputs are velocities). The interest of this study mainly lies in the fact that it shows how to characterize the controllability properties by switching between the modes $S_{i}$ of the associated automaton. The natural notion of controllability restricted to constraint surfaces of the configuration space is also introduced in [38]. The previous studies use the geometric structure of nonsmooth mechanical systems [24].

OP 8: Depending on the input matrix $E$ in (3), the unconstrained system may not be controllable [e.g., underactuated systems with $l<n / 2$, or the structure in (20)]. Can the addition of complementarity conditions and state reinitialization improve its controllability properties? So what is the relation between the controllable subspaces of the dynamics in each mode, the state reinitialization rule and the set of admissible controls $\mathcal{U}$, so that the CS is controllable? A path for local controllability study may be to use the linearization of the solution in (19) which incorporates the so-called saltation matrix [27].
Some preliminary answers have been given for juggling systems [58], [16], [17], [94], which are a subclass of mechanical $\mathrm{CS}$ as in (3) that possess the following general form:

$$
\left\{\begin{array}{l}
\dot{z}_{1}=f_{1}\left(z_{1}, t, \lambda\right) \\
\dot{z}_{2}=f_{2}\left(z_{2}, u, \lambda\right) \\
0 \leq y=h\left(q_{1}, q_{2}\right) \perp \lambda \geq 0 \\
\text { Collision rule }
\end{array}\right.
$$

with $z_{i}^{T}=\left(q_{i}^{T}, \dot{q}_{i}^{T}\right) \in \mathbb{R}^{n_{i}}$. Examples of mechanical jugglers are running biped robots, hoppers, controlled structures, nonprehensile manipulation systems, manipulators with dynamic passive environment, systems with dynamic backlash or liquid slosh phenomena [60], tethered satellites [52], etc. Provided the $z_{2}$ dynamics is controllable, the unconstrained system with $\lambda=0$ is therefore written in an uncontrollable canonical form. The only way to control $z_{1}$ is through the collisions, i.e., by using $\lambda$ as an input. The basic idea in [16] and [17] is to study the controllability properties of a partial impact Poincaré map $P_{\Sigma}$ with state $\dot{q}_{1}(k) \triangleq \dot{q}_{1}\left(t_{k}^{-}\right)$and input $\dot{q}_{2}(k) \triangleq \dot{q}_{2}\left(t_{k}^{-}\right)$ (see, e.g., [16, Lemma 3]). Reachable sets and accessibility are defined accordingly. The Poincaré section is chosen as $\Sigma=\left\{(q, \dot{q}): h(q)=0, \nabla h(q)^{T} \dot{q}>0\right\}, q^{T}=\left(q_{1}^{T}, q_{2}^{T}\right)$. This controllability problem may be stated as follows [17].

Problem 1: Given $\left(q_{1}^{1}, v_{1}^{+}\right)$with $v_{1}^{+} \neq 0,\left(q_{1}^{n}, v_{n}^{+}\right)$and $n \geq 2$, find $\left\{q_{1}(k)\right\}_{2 \leq k \leq n},\left\{\dot{q}_{1}(k)\right\}_{2 \leq k \leq n},\left\{q_{2}(k)\right\}_{1 \leq k \leq n}$ and $\left\{\dot{q}_{2}(k)\right\}_{2 \leq k \leq n}$, such that $\left(q_{1}\left(t_{1}\right), \dot{q}_{1}\left(t_{1}^{+}\right)\right)=\left(q_{1}^{1}, v_{1}^{+}\right)$and $\left(q_{1}\left(t_{n}\right), \dot{q}_{1}\left(t_{n}^{+}\right)\right)=\left(q_{1}^{n}, v_{n}^{+}\right)$.

In other words, one looks for a sequence of impacts to join the initial and end points. The locations, velocities, and number of impacts, are unknown of the problem. Denote $x_{1}^{T}=\left(q_{1}^{T}(2), \ldots, q_{1}^{T}(n-1)\right), x_{2}^{T}=\left(q_{2}^{T}(1), \ldots, q_{2}^{T}(n)\right)$, $x_{3}^{T}=\left(\dot{q}_{1}^{T}(2), \ldots, \dot{q}_{1}^{T}(n-1)\right), x_{4}^{T}=\left(\dot{q}_{2}^{T}(2), \ldots, \dot{q}_{2}^{T}(n)\right)$. Let us denote $x_{4}$ as an input vector $w \in \mathbb{R}^{n-1}$, and $x^{T}=\left(x_{1}^{T}, x_{2}^{T}, w^{T}\right)$. As shown in [17] when the vector fields $f_{1}(\cdot), f_{2}(\cdot)$ and $h(\cdot)$ in (20) possess some linearity, finding a solution to problem 1 is equivalent to finding a solution to the constrained equation

$$
\left\{\begin{array}{l}
\mathcal{M}\left(w, q_{1}^{1}, v_{1}^{+}\right)\left(\begin{array}{c}
q_{n}^{1} \\
v_{n}^{+}
\end{array}\right)+\mathcal{N}\left(x, q_{1}^{1}, v_{1}^{+}\right)=0 \\
\mathcal{P}\left(w, q_{1}^{1}, v_{1}^{+}\right) q_{n}^{1}+\mathcal{T}\left(x_{1}, w, q_{1}^{1}, v_{1}^{+}\right) \geq 0
\end{array}\right.
$$

where the matrices $\mathcal{M}, \mathcal{N}, \mathcal{P}$ and $\mathcal{T}$ depend nonlinearly on the initial state $\left(q_{1}^{1}, v_{1}^{+}\right)$and on $x$. If (21) possesses a solution $x$, then $\left(q_{1}^{n}, v_{n}^{+}\right)$is reachable from $\left(q_{1}^{1}, v_{1}^{+}\right)$. The example of an impacting pair (modeling dynamic backlash [60]) is treated in [17], and some general results are given which make use of the particular structure of the constrained equation in (21) and of CP constructed from the Karush-Kuhn-Tucker conditions [74]. Comparing the constrained equation in (21) with the linear system of equations that characterizes the controllability of discrete-time invariant linear systems [93, Sec. 3.2], shows how nonlinear jugglers are.

OP 9: Investigate the structure of the reachable sets. How may one use tools from linear and nonlinear programming to get a general criterion on accessibility of jugglers? Can local controllability criteria be set up, and if so are they always relevant? Indeed in certain cases global criteria may be needed. 
It is particularly interesting to note that jugglers may not be locally accessible, but only globally accessible [17], [58]. In other words, problem 1 may not possess any solution for $\left(q_{1}^{n}, v_{n}^{+}\right)$and all intermediate locations and velocities lying in a neighborhood of $\left(q_{1}^{1}, v_{1}^{+}\right)$. This is the case of a planar juggler $\left(n_{1}=4, n_{2}=2\right)$ whose reachable sets have been fully characterized in [58].

OP 10: Characterize the controllability of (20) from $u$. The major ingredients are: the controllability of the $x_{2}$-dynamics, the controllability of the $x_{1}$-dynamics in the sense of problem 1 , the invertibility of the collision mapping. Use the aforementioned framework to study the influence of various characteristic physical of systemical constants (restitution coefficient, Darboux-Keller shock dynamics [12, Sec. 4.2.5], [96], relative degrees between $y, u, \lambda$, etc.) on the controllability.

\section{Affine Complementarity Systems}

1) General Considerations: Let us consider the ACS in (13). It is crucial to note that the slack variable $\lambda$ is not an exogenous disturbance, so even when a matching condition between $u$ and $\lambda$ is satisfied, one may not compensate for $\lambda$ directly with $u$. The switches between the modes $S_{i}$ play a crucial role. If $u(t)$ is a function, instantaneous switches at arbitrary time $t$ are impossible in general. In other words, in most CS the event times $t_{i, j}$ are not directly controllable.

OP 11: Is it possible to include distributional inputs in $\mathcal{U}$, whose action would be similar to a discrete control by taking instantaneously the state $x(t)$ from one mode to another one?

As we shall see in Section V-D-2, in some cases of ACS, bounded discontinuous inputs $u(\cdot)$ can act as discrete controls and help invert some nonsmooth characteristics. The application of distributional inputs $u(\cdot)$ (e.g., Dirac measures and derivatives) to a CS, certainly requires much care. Until now we have only considered state jumps which are ruled by a reinitialization mapping. Applying distributional inputs to ACS [even if solutions are $\mathrm{AC}$ for all bounded $u(\cdot)$ ] certainly is a delicate matter in general since they become complex MDE (see Section IV). The case where both reinitialization mapping and distributional input coexist, is by far not yet well understood. Notice that such questions may be fundamental when dealing with optimal control problems.

Remark 6: Consider example 8. The dynamics in (11) can be rewritten as a first-order sweeping process [53]

$$
-\dot{x} \in \partial \psi_{\mathbf{R}^{+}}(x+u)=\partial \psi_{[-u(t),+\infty)}(x) .
$$

The right-hand side of the inclusion in (22) is noncompact and depends on both $u$ and $x$, whereas in (16) it is compact and depends on the state only, and in (4) it is noncompact and depends on the state only. Which are the consequences of these properties on controllability? It is important to remark here that the nature of $u(t)$ (continuous, piecewise continuous, RCLBV function) influences much the nature of $x(t)$ in the sweeping process. If $u(\cdot)$ is Lipschitz, then $x(\cdot)$ is Lipschitz. If $u(\cdot)$ is RCLBV then $x(\cdot)$ is RCLBV [53]. Therefore, controllability may be more difficult to study, since modifying the input space implies modifying the solutions as well (especially introducing jumps).

OP 12: The controllability of the ACS in (13) with $G=-I_{m}$ and $\mathrm{AC}$ solutions may be attacked as follows. Assume that $E$ is full-column rank and let $E^{\dagger} E=I_{m}, W E=0_{(n-m) \times m}$. Then, one obtains

$$
0 \leq-E^{\dagger}(-\dot{x}+A x+B u+\bar{e}) \perp \bar{C} x+\bar{F} u+\overline{\bar{d}} \geq 0
$$

where $\bar{C} x+\bar{F} u+\overline{\bar{d}}=C x+F u+D \bar{\lambda}+\bar{d}$ and $\bar{\lambda}$ is the solution of the LCP: $0 \leq y \perp \lambda \geq 0$, and

$$
W(-\dot{x}+A x+B u+\bar{e})=0 .
$$

How may one use the $\mathrm{CP}$ in (23) and the reduced-order dynamics in (24) (that may be a mixed or a simple LCP in $u$ ) for controllability purpose? Can one formulate similar conditions as (23) and (24) from a time-discretization of (14) and then deduce some properties of the continuous-time system by studying the limit of the discrete-time solutions [59], [63]? What is the influence of the convergence (strong, weak, weak $\star$ [5], [95], [90]) on such a study? Is it possible to formulate some verification or feasibility criterion [6] that would allow the designer to test numerically some approximate controllability properties from a time-discretization?

It is known that mere LCPs may be quite simple or almost untractable problems [8], depending on the properties of the LCP matrix. This is expected to impose severe restrictions on the data in (23) and (24) to get solutions of OP 12.

OP 13: Since $u$ and $\lambda$ can be seen as an exogenous and a state (and $u$ ) dependent inputs, respectively, what is the role of the pairs $(A, B),(A, E)$ and $(F, C)$ in the controllability of (13)?

The study of simple first-order examples as in (2), with the input acting both in $y$ and in the vector field $f(\cdot)$, might constitute a good starting point.

OP 14: Let us consider an ACS in (13) with $\bar{e}=0, \bar{d}=0$, $G=-I_{m}, F=0, D=0$. Then from (14) its DI formalism reads $-\dot{x} \in\{A x\}+E \partial \psi_{\left(\mathbb{R}^{+}\right)^{m}}(C x)+\{B u\}$. Mimicking linear invariant systems [93], its conjugate (or adjoint) system may be defined as $-\dot{\xi} \in G(\xi), z=B^{T} x$, where $G(\xi)$ is the conjugate of $A x+E \partial \psi_{\left(\mathbb{R}^{+}\right)^{m}}(C x)$. How is controllability of the $x$ system related to observability of the $\xi$ system?

2) Inversion of Piecewise Linear Characteristics: The inversion of the operator $\mathrm{PL}(\cdot), u_{2}=\operatorname{PL}\left(z_{1}\right)$, whose graph is as in Fig. 1 is certainly an important property for the controllability of the overall system. The problem here is to find a $u$ such that PL $(u)=v_{d}$ for some desired signal $v_{d}$. The deadzone of Fig. 3(b) with $a=b>0$ is invertible on $\mathbb{R}$ : let us denote its output as $v$ and let us consider $u(t)=(1 / a) v_{d}(t)+d \operatorname{sgn}\left(v_{d}(t)\right)$, $\operatorname{sgn}(\cdot)=\partial|\cdot|$. Then $v(t)=v_{d}(t)$ for all $t \geq t_{0}$. Actually, one sees that the graph of PL: $v_{d} \mapsto u$ is exactly the inverse graph [86, p. 219] of the deadzone graph. The trick is that such a $u$ allows the system to jump between modes $S_{2}$ and $S_{3}$, avoiding mode $S_{1}$ : it acts as a discrete control. This is closely linked to having $F \neq 0$ in (13), see (7). One sees that the complementarity formalism clearly shows why some nonlinearities are invertible: $u(\cdot)$ directly acts inside the complementarity conditions and allows one to impose switches of $\lambda$.

OP 15: Determine the conditions on the subsystem $H_{1}(s)$ in Fig. 1 and/or the set of admissible inputs $\mathcal{U}$ such that invertibility of PL implies controllability of the overall system.

In conclusion, the controllability of CS as in (1) is a difficult problem because they possess no discrete control, and 
steering trajectories $x(\cdot)$ with the input $u(\cdot)$ involves an interplay between the continuous dynamics and complementarity conditions.

\section{Stabilization AND FEedBack CONTROL}

Consider for instance an ACS $\dot{x}=A x+C^{T} \lambda, 0 \leq y=$ $C x+\bar{d} \perp \lambda \geq 0$. As shown in [75, ex. 3.2], the stability of the system $\dot{x}=A x$ does not imply that of this ACS [which is an evolution variational inequality (EVI)]. Stabilization of CS is therefore a subject that requires full attention. The characterization of invariant sets is a prerequisite to stabilization. The first section provides some insights on fixed points calculation for CS. Then we discuss stability, dissipativity, and optimal control. Finally the tracking control of two classes of CS (Lagrangian systems and jugglers) is briefly reviewed.

\section{A. Existence of Fixed Points}

Let us consider the system in (4) with $u=0$. Then the point $\left(q(t), \dot{q}\left(t^{+}\right)\right)=\left(q_{0}, 0\right)$ is a fixed point of this MDI if and only if the generalized equation (see Appendix B)

$$
0 \in \partial \psi_{\Phi}\left(q_{0}\right)+\frac{\partial U}{\partial q}\left(q_{0}\right)
$$

holds. The function $U(q)$ is the potential energy of the system and $F(q, \dot{q})=C(q, \dot{q}) \dot{q}+(\partial U / \partial q)$, where $C(q, \dot{q}) \dot{q}$ denotes Coriolis and centripetal torques. We also used the fact that $\left(\dot{q}\left(t_{k}^{-}\right)=0\right) \Rightarrow\left(\dot{q}\left(t_{k}^{+}\right)=0\right)$ in the impact law, since $0 \in V(q)$. Let us notice that the condition (25) does not imply at all the uniqueness of the fixed point (for instance systems with clearance in which there is an infinity of fixed points [60]).

Example 10: Let us consider the simple example

$$
m \ddot{q}=-m g, \quad q \geq \delta>0 \quad \dot{q}\left(t_{k}^{+}\right)=-e \dot{q}\left(t_{k}^{-}\right) .
$$

Obviously $\left(q, \dot{q}\left(t^{+}\right)\right)=(\delta, 0)$ is the unique fixed point and one can check that condition (25) is satisfied. The same dynamics with $q \leq 0$ no longer possesses any fixed point.

Lemma 1: Assume that in (3) one has $q \in \mathbb{R}^{n}, h(q)=$ $C q, C \in \mathbb{R}^{m \times n}, m<n, F(q, \dot{q})=C(q, \dot{q}) \dot{q}-Q+K q$, $Q \in \mathbb{R}^{n}$ and $K \in \mathbb{R}^{n \times n}$ constant, and $C$ is full row rank. Then equilibrium points $q_{0}$ satisfy the Extended $\operatorname{LCP}(q): 0 \leq$ $C^{\dagger}(K q-Q-E u) \perp C q \geq 0$ and $W(Q-K q+E u)=0$, where $C^{\dagger}=\left(C C^{T}\right)^{-1} C \in \mathbb{R}^{m \times n}$ and $W C^{T}=0, W \in$ $\mathbb{R}^{(n-m) \times n}$.

The proof proceeds as follows. The equilibrium $(q, \dot{q})=$ $\left(q_{0}, 0\right)$ has to satisfy the generalized equation $Q-K q+E u \in$ $C^{T} \partial \psi_{\left(\mathbb{R}^{+}\right)^{m}}(C q)$, since $0 \leq C q \perp \lambda \geq 0$. Hence, multiplying both sides of this generalized equation by $C^{\dagger}$ and $W$ one gets $C^{\dagger}(Q+E u-K q) \in \partial \psi_{\left(\mathbb{R}^{+}\right)^{m}}(C q)$ and $W(Q-$ $K q+E u)=0$. The first generalized equation is a LCP: $0 \leq$ $C q \perp-C^{\dagger}(Q-K q+E u) \geq 0$, and the second condition is the equality $W Q+W E u=W K q$. This can be written as the ELCP (see Appendix B): $A q-d \geq 0, B q=e, \sum_{j=1}^{m} \prod_{i \in \phi_{j}}(A q-$ $d)_{i}=0, \phi_{j}=\{j, m+j\}$, with $A=\left(\begin{array}{c}C \\ C^{\dagger} K\end{array}\right) \in \mathbb{R}^{2 m \times n}$, $d=\left(\begin{array}{c}0 \\ C^{\dagger}(Q+E u)\end{array}\right) \in \mathbb{R}^{m \times n}, B=W K, e=W Q+W E u$. Algorithms to solve ELCPs exist [92]. The results in [36] may also be used to study and solve (25) (if $K=K^{T}>0$ then this ELCP has one solution from [36, Th. 2.2]).
Generally, the fixed points of CS are the solutions of generalized equations $0 \in F(x)$.

\section{B. Dissipative Systems}

1) Lagrange-Dirichlet Theorem: It is crucial to develop a stability analysis framework for systems as in (1). For Lagrangian mechanical systems as in (3), the mathematical results of [95] and [5] provide a nice ground. In summary, they imply (under some conditions which are not recalled here) the following.

a) $q \in A C, \dot{q} \in R C L B V$ (hence any quadratic function of $\dot{q}$ is RCLBV as well [63]).

b) Therefore, the distributional derivative $d \ddot{q}$ can be decomposed as the sum of three terms: an atomic measure $d \mu_{a}$, a Lebesgue integrable function $\{\dot{q}\}$, and a nonatomic measure singular with respect to the Lebesgue measure $d \mu_{n a}$.

c) The set of impact times $\left\{t_{k}\right\}_{k \geq 0}$ is countable, and there exist possible left-accumulations $\lim _{k \rightarrow+\infty}=t_{\infty}<$ $+\infty$.

These very specific features are at the core of the developments in [12], [13], [11], [18], and [101]. Item b) is crucial, since it means that despite the time derivative of a Lyapunov function $V \in R C L B V$ is not a function, it is a measure. Consequently, characterizing the sign of $d V$ makes perfect sense, and if the measure $d V \leq 0$, the function $V$ is decreasing [29] (in other words, RCLBV functions are the primitive of their distributional derivative). Such conclusions would not have been possible if $\dot{q}$ had been proved to be measurable only. Item c) secures that the set $\left\{t_{k}\right\}_{k \geq 0}$ is an admissible event times set [21, Def. 3.3.4]. The measure $d \mu$ in (5) can be chosen as $d \mu=d t+d \mu_{a}$. We note that without Coulomb friction and if all data are piecewise analytic, then $d \mu_{n a}=0$ [5]. It is noteworthy that the fact that velocities are RCLBV does not preclude some very complicated phenomena like accumulations or accumulations of impacts (concretely, let $\left\{t_{k}^{1}\right\}$ and $\left\{t_{k}^{2}\right\}$ be two sequences of impacts with limits $t_{\infty}^{1}$ and $t_{\infty}^{2}$, respectively, and let $t_{\infty}^{1}$ tend toward $t_{\infty}^{2}$ so that both accumulations are merged into a single one). So far, it has not been proved that such phenomena do not occur in mechanical systems with unilateral constraints and nonpurely elastic collisions.

Lemma 2: Consider a mechanical system as in (5) where $F(q, \dot{q})$ consists of Coriolis, centripetal and conservative generalized forces with a smooth potential $U(q), u=0$. Then, if $\psi_{\Phi}(q)+U(q)$ has a strict minimum at $q_{0}$, the equilibrium point $\left(q_{0}, 0\right)$ satisfying $(25)$ is stable in the sense of Lyapunov.

Items a) and b) are in force here. The proof [18] is based on the use of the nonsmooth Lyapunov candidate function

$$
V(q, \dot{q})=\frac{1}{2} \dot{q}^{T} M(q) \dot{q}+\psi_{\Phi}(q)+U(q)-U\left(q_{0}\right)
$$

and on basic nonsmooth and convex analysis. Since $q(t) \in \Phi$ for all $t \geq \tau_{0}$, it follows that $V(t) \in \mathrm{RCLBV}$ and one has along trajectories of (5)

$$
\begin{aligned}
\frac{d V}{d \mu}=\left[\dot{q}\left(t^{+}\right)+\right. & \left.\dot{q}\left(t^{-}\right)\right]^{T} M(q(t)) \frac{d v}{d \mu}+\dot{q}\left(t^{+}\right)^{T} z \frac{d t}{d \mu}+\frac{\partial U}{\partial q} \frac{d q}{d \mu} \\
& +\frac{1}{2} \frac{\partial}{\partial q}\left(\dot{q}\left(t^{+}\right)^{T} M(q(t)) \dot{q}\left(t^{+}\right)\right) \frac{d q}{d \mu} \leq 0
\end{aligned}
$$


where $z \in \partial \psi_{\Phi}(q(t))$ and Moreau's rule for differentiation of quadratic function of RCLBV functions [63] has been used. The advantage of using the MDI formalism, is that it might be a powerful tool in studying systems such that the measure $d \mu$ is more complex [e.g., stochastic friction models inherently containing nonzero measures $d \mu_{n a}$ as in item b), or such that the support of $d \mu_{a}$ is not contained in $\left.\{t \mid q(t) \in \partial \Phi\}\right]$. It is of primary importance to note that the mapping $\dot{q}\left(t^{+}\right) \mapsto \partial \psi_{V(q(t))}\left(\dot{q}\left(t^{+}\right)\right)$in (5) is monotone, since $V(q)$ and $\partial \psi_{V(q(t))}(\cdot)$ are polar cones. Lemma 2 brings an answer to whether or not the system

$$
\left\{\begin{array}{l}
M \ddot{q}+F \dot{q}+K q+G=C^{T} \lambda \\
C q \geq 0, \quad \lambda \geq 0 \quad \lambda^{T} C q=0 \\
\text { Collision mapping }
\end{array}\right.
$$

where $M, C, K, G, F$ are constant, has bounded solutions for all $t \geq 0$. The reader may check its application on simple systems like one degree of freedom jugglers [16], impacting pair modeling backlash [60], with collocated PD feedback controllers.

OP 16: Extend Lemma 2 to systems with unilateral constraints and Coulomb friction, with $\Phi$ nonconvex and $m \geq 2$. Extend it to all mechanical systems subject to set valued force laws that derive from a superpotential.

It is likely that solving OP 16 requires first modeling investigations [33], [35].

2) Absolute Stability: Let us now turn our attention to a class of ACS. The absolute stability problem (see, e.g., [57]) consists of studying the stability of a the negative feedback interconnection of $H(s)$ (a positive real transfer function $u \mapsto y$ ) with a sector static nonlinearity $\phi(t, y)$ with slope in $[0,+\infty)$. Usually, $\phi(\cdot, \cdot)$ is required to be piecewise continuous in $t$ and locally Lipschitz in $y$. It is of interest to extend this result to the case where the feedback nonlinearity is a maximal monotone multivalued mapping.

A multivalued mapping $F(\cdot)$ is monotone if $\left(x-x^{\prime}\right)^{T}(y-$ $\left.y^{\prime}\right) \geq 0$ for any couples $(x, y)$ and $\left(x^{\prime}, y^{\prime}\right)$ in its graph. Monotone mappings correspond to completely nondecreasing curves in $\mathbb{R}^{2}$. Maximality of multivalued monotone operators refers to graph inclusion (i.e., the gaps are filled in). The relay function defined as $u_{2}=R L\left(z_{1}\right), u_{2} \in\{-1,1\}$ if $z_{1}=0$, is not maximal. However, the graphs in Fig. 3(e), $(f)$, and (h) are maximal monotone.

The considered state-space equations are

$$
\left\{\begin{array}{l}
\dot{x} \stackrel{\text { a.e. }}{=} A x+B w \\
z=C x \\
-w \in \partial \varphi(z)
\end{array}\right.
$$

where a.e. is almost everywhere because solutions are expected to be AC. The link with CS is clear (choose, e.g., $\left.\varphi(\cdot)=\psi_{\left(\mathbf{R}^{+}\right)^{m}}(\cdot), \operatorname{dim}(z)=m\right)$. The system in (16) fits within (30) with $x=\dot{q}$ and $\varphi(\cdot)=|\cdot|$, as well as $\left(S_{1}\right)$ in (2) with $a=0$, see (17). The fixed points of (30) can be characterized as in Section VI-A. Let us assume that $H(s)=C(s I-A)^{-1} B$ is a strictly positive real transfer function [57], that $\varphi(\cdot)$ is lsc and convex, so that $\partial \varphi(\cdot)$ is a maximal monotone multivalued mapping [86], and that the graph of $\partial \varphi(\cdot)$ contains $(0,0)$.
Lemma 3 [18]: The solutions $x(\cdot)$ of (30) satisfying $C x(0) \in \operatorname{dom} \partial \varphi$ are $\mathrm{AC}$ and uniqueness holds. The fixed point $x=0$ of the system in (30) is unique and is globally asymptotically stable.

The stability proof relies on a quadratic Lyapunov function $V(x)=(1 / 2) x^{T} P x$ with $P$ the solution of the Kalman-Yakubovic-Popov lemma conditions [57]. Related results can be found in [20] (passive LCS) and in [75, Ch. 3] (evolution VI). Passive LCS have the property that the transfer function $\lambda \mapsto y$ is positive real. Passifiability by pole shifting is an interesting result for stabilization by feedback [20]. The well-posedness proof of Lemma 3 requires some manipulations and the fixed point is the solution of the generalized equation $0 \in A x-B \partial \varphi(C x)$. It seems that Brézis' theorem on global existence and uniqueness of solutions [10] to DI $-\dot{x} \in A(x)$, $A(x)$ a maximal monotone operator, is central in the study of interconnections as in (30). The PR condition on $(A, B, C)$ can be relaxed when $\varphi(\cdot)=|\cdot|$ [83], at the price of restricting the set of solutions to piecewise differentiable functions. Let us notice that (30) is a particular DI. Many extensions exist, e.g., considering $z=C(x(t))$, where $C(\cdot)$ is a monotone operator. When $A=0$ and $B$ the identity, (30) corresponds to the DI studied in [50]. Therefore, infinite dimensional generalizations of lemma 3 should be possible.

OP 17: A next step is also to extend Lemmas 2 and 3 toward ACS as in (13). Then, extend toward dissipative nonlinear dynamics $\dot{x}=f(x)+g(x) w, z=h(x)$.

Actually, Lemma 3 readily extends to nonlinear systems of the form $\dot{x}=-\sum_{k=0}^{N} x^{2 k+1}+w, z=x,-w \in \partial \varphi(z)$, $x \in \mathbb{R}$, with storage function $V(x)=x^{2} / 2$. The operator $w \mapsto z$ is strictly passive with dissipation function $S(x)=$ $-\sum_{k=0}^{N} x^{2 k+2}$ [57].

Remark 7: The following is an issue [12], [20]: Since the supply rate $W(u, y)=y^{T} \lambda$ may be meaningless in the sense of Schwarz' distributions at times $t_{k}$, how to extend the dissipation equality (integrated or infinitesimal forms) to CS? Possible paths have been pointed out in [12, Sec. 1.2.2].

Finally, a natural extension of lemmas 2 and 3 is:

OP 18: Characterize classes of CS such that the Krakovskii-LaSalle invariance lemma applies.

The invariance lemma relies on properties of the positive limit sets (compactness, invariance) which in turn are a consequence of properties of solutions like uniqueness, continuous dependence in the initial data, as well as properties of the Lyapunov function itself. By far, not all CS meet all these requirements.

\section{Optimal Control}

Let us focus on mechanical systems as in (3), to which items a), b), and c) apply. As pointed out in [12, Sec. 3.5.4] the following problem remains unsolved.

OP 19: Find $u^{\star}$ that is critical for some action integral $I(u)=\int L(q, \dot{q}, t) d t+\beta(x(T), T)$, under the dynamics $\ddot{q}=u+\lambda, 0 \leq q \perp \lambda \geq 0, \dot{q}\left(t_{k}^{+}\right)=-e \dot{q}\left(t_{k}^{-}\right)$. How do the number of impacts and the restitution influence $I(u)$ ? In which space $\mathcal{U}$ should one consider $u$ ? Then generalize to higher order systems.

Applications are in the optimal control of all mechanical systems subject to impacts and unilateral constraints. It is note- 
worthy that the optimal control of MDE (see, e.g., [91]), does not solve OP 19. The optimal control of a time invariant linear system with virtual state constraints $C x \geq 0$ yields optimality conditions [87]

$$
\left\{\begin{array}{l}
\dot{z}=\bar{A} z+\bar{B} \lambda \\
0 \leq \bar{C} z \perp \lambda \geq 0
\end{array}\right.
$$

for some $z$ and matrices $\bar{A}, \bar{B}, \bar{C}$. Notice the striking similarity between (31) and (6), though both systems represent quite different problems.

A prerequisite to a better understanding of optimal control for the aforementioned classes of systems, is a better understanding of the dynamics of CS. The optimal control of a simple ACS has been solved in [23, Sec. 5.3] and extensions toward more general ACS may be an objective. Applications are in optimal control of electrical networks with diodes and/or MOS transistors.

\section{Tracking Control for Lagrangian Systems}

This problem has been studied in [13], [11], and [62], for systems as in (3), with a single constraint, and $E$ the identity matrix. In [62], planar systems inside a disc are considered, with elastic impacts. PD inputs are shown to asymptotically stabilize particular impacting trajectories. The objective in [13] and [11] is to design a controller that assures stable tracking of some reference trajectory $q_{d}(t)$, relying on a suitable stability framework that encompasses both unconstrained, persistently constrained, and impacting orbits. Features a), b), and c) enumerated in the introduction of Section VI-B hold, and the goal is to extend lemma 2 to the case of tracking control. There are four main features in this extension.

i) Impacts have to be included in any stability analysis, and the designer should take advantage that impacts imply a loss of kinetic energy in the stabilization.

ii) There are two antagonist facts: robustness of the stabilization process on $\partial \Phi$ requires that a "bouncing-ball"like dynamics be realized in closed loop [notice that $\delta$ in (26) needs not to be known to get stabilization], but asymptotic stability implies that velocity jumps vanish asymptotically.

iii) Requiring that $\{\dot{V}\} \leq 0$ for all $t \geq 0$ [see item b)] is too stringent in most cases, especially during transition phases including rebounds.

iv) The underlying nature of the evolution process and the trajectories has to be taken into account.

Item i) leads to the choice of a Lyapunov function which is as close as possible to the process total energy. The function $V(t, \tilde{q}, \dot{\tilde{q}})=(1 / 2) \dot{\tilde{q}}^{T} M(q) \dot{\tilde{q}}+\psi_{\Phi}(q)+\tilde{U}(\tilde{q})-\tilde{U}\left(\tilde{q}_{0}\right)$ is a suitable one, $\tilde{q}=q-q_{d}$. Item ii) implies a specific transition phase in order to stabilize the system on $\partial \Phi$. The dynamics should be similar to (26) during the transient, whereas a tangent approach should be asymptotically guaranteed. Attaining the constraint tangentially, and without incorporating impacts in the stability analysis, cannot work neither theoretically nor in practice. The main source of difficulty for stability lies in the coupling between normal and tangential directions to $\partial \Phi$, i.e., the $M(q)$ generally cannot be transformed in a block-diagonal matrix via a suitable generalized coordinate change. When the

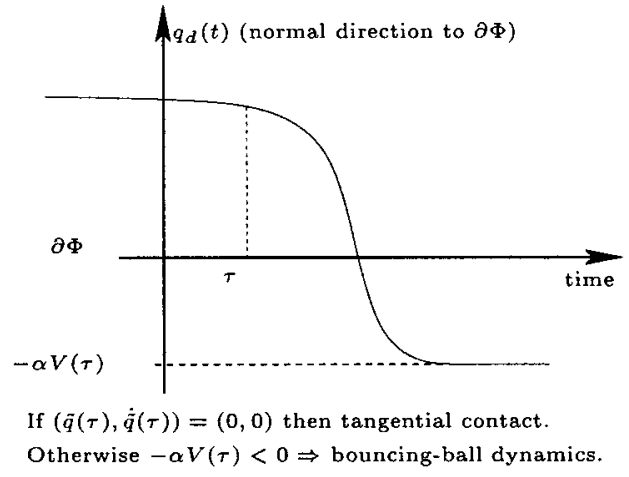

Fig. 5. Transition phase trajectory.

dynamics is decoupled, the control problem is much easier [12, Sec. 8.6.3]. Item iii) means that it is often more convenient to think of the dynamics during the transition phase, in terms of an impact Poincaré map $P_{\Sigma}$ with Poincaré section $\Sigma$ as in problem 1. Item c) secures that such a discrete-time system can be constructed. Then stability may be characterized as the Lyapunov stability of $P_{\Sigma}$ with Lyapunov function the restriction of $V$ to $\Sigma^{-}$. Item iv) means that the dynamics in (26) and

$$
m \ddot{\tilde{q}}+\gamma_{1} \dot{\tilde{q}}+\gamma_{2} \tilde{q} \in-\partial \psi_{\mathbf{R}^{+}}(q), \quad q_{d}(t)>0 \quad \forall t \geq 0
$$

are of different natures. In (26), the invariant set is the solution of the generalized equation (25) and is, therefore, characterized by all the ingredients of the dynamics (including the impact law). In (32), the invariance is simply that of the continuous dynamics, since $\partial \psi_{\mathbf{R}^{+}}\left(q_{d}(\cdot)\right)=\{0\}$. The stability framework and the controller proposed in [11] and [13] take these peculiar features into account. The proposed controller is based on the Paden and Panja controller [57, Sec. 6.2.5] for unconstrained systems. In particular the transition phase uses a signal $q_{d}(t)$ as depicted in Fig. 5, with a closed-loop dynamics similar to (32) in the normal direction to $\partial \Phi$, to cope with item ii). The design of $q_{d}(\cdot)$ during the stabilization phase on $\partial \Phi$ is a crucial step. The use of the (fixed parameter) Slotine and Li controller might be of interest too in this context [101], since it possesses the advantage over the Paden and Panja's one of assuring $\dot{V}(t) \leq-\gamma V(t), \gamma>0$, on free motion phases. However, the function $V(\cdot)$ is less close to the mechanical energy for this controller. In fact, Moreau's MDI in (5) has not yet been fully exploited in this setting.

The tracking problem for fully actuated complementarity Lagrangian systems is not a simple extension of the unconstrained case. The design of a suitable desired trajectory during transition phases, is a crucial step.

The aforementioned results apply to the case $m=1$, i.e., a unique constraint with $h(q)$ in (3) continuously differentiable. Let $\Sigma_{i}=\left\{q \mid h_{i}(q)=0\right\}$.

OP 20: Study control strategies that stabilize the system on the codimension $2 \leq p \leq m$ surface $\Sigma_{p}=\Sigma_{1} \cap \Sigma_{2} \cap \cdots \cap \Sigma_{p}$, with any initial conditions. In particular, the transition phase controller should be robust with respect to all types of uncertainties that may modify some dynamical properties. Then extend the tracking controllers of [11] and [13] which concern (3), to the case $m \geq 2$. 
The impact with the surface $\Sigma_{p}$ is called a multiple impact. Let us recall that Lyapunov stability is equivalent to some continuity of solutions with respect to initial data, on an infinite time interval. Multiple impacts may destroy this property, see [42], [90], [12]. This depends a lot on the domain $\Phi$ geometry, the mass matrix $M(q)$ and the impact law. Therefore uncertainties on these physical data may drastically modify the trajectories which collide in the neighborhood of $\Sigma_{p}$. Once OP 20 has been solved, proceed to the following.

OP 21: Extend the results in [13] and [11] to the case of joint flexibilities.

Both OP 20 and 21 have important practical consequences. Indeed in most mechanical systems $m \geq 2$. Concerning flexibilities, it is expected that the effect of vibrations in the structure due to the impacts, may be modeled this way. The consequences on stabilization may be crucial. The control of biped robots in a walking motion seems quite particular and its link with OP 20 needs to be clarified. Indeed the basic stability of a walking biped requires that at each instant the state and the input satisfy inequalities $A(q) u+B(q, \dot{q})>0$ outside impacts and $\left.\bar{A}\left(q\left(t_{k}\right)\right) \dot{q}\left(t_{k}^{-}\right)\right)>0$ at impacts [46], for some matrices and vectors $A, B$ and $\bar{A}$. Designing such a feedback control is an open problem. The extension toward higher order CS as $\left(S_{3}\right)$ in (2) is challenging as well.

Control of Jugglers: Quite interesting stabilization results have been obtained in [58] for a planar juggler, using ideas of control recurrence. A framework for the control design of mechanical systems as in (20) has been proposed in [16], and the control of an impacting pair modeling dynamic backlash has been studied in [60]. A feedback stabilization technique using the linearization of (19) is used in [27]. An interesting problem is in the control of buildings subject to earthquake excitations, modeled with the rocking block approach [12]; where should the actuators be placed so as to avoid overturning, and minimize the angular motion magnitude? This is thought to be a tough problem (including multiple impact and friction).

OP 22: Characterize classes of tasks in terms of the closed-loop invariant sets to be stabilized, and derive a general stability and controller design framework.

\section{CONCLUSION}

The class of nonlinear nonsmooth controlled dynamical systems considered in this paper (namely CS) is quite interesting, since it still offers many challenges to various scientific communities (including systems and control) and at the same time finds many applications. Such systems may be recast in the class of hybrid dynamical systems, but can also be seen as measure differential inclusions. The adopted formalism depends on the objective of the study. The goal of this paper is to expose the difficulties and main problems associated to such complex dynamics. Several notions which are peculiar to complementarity dynamical systems are introduced, such as sets of admissible states and inputs, normal controllability, viability of controls. One interesting question is whether it is possible to classify complementarity dynamical systems into subclasses with specific control properties, or not. Until now, the studies have focused on linear CS [20], [21], [40]-[42], [89], nonlinear CS [88] and mechanical systems [11]-[13], [16], [45], [56], [58], [62],
[61], [76], [82]. Complementarity dynamical systems do represent a very specific class of hybrid systems for which many problems heavily rely on the use of complementarity problems and convex analysis. This peculiar structure is very useful since it allows one to deeply investigate their properties, and at the same time is not restrictive in terms of potential applications which are numerous. The possible relationships with other types of nonsmooth evolution problems are pointed out. Some open problems are proposed; clearly many others exist and will hopefully be given solutions. Finally, it is worth noting that the numerical simulation of complementarity systems is also a hard problem and that most available software packages do not allow to treat correctly neither complementarity problems nor finite accumulation of events [15].

\section{APPENDIX A}

\section{MEASURE DIFFERENTIAL INCLUSIONS}

The classical equation of dynamics is an equality of functions, like $m\left(d^{2} q / d t^{2}\right)(t)=f(t)$. Imagine now that the velocity has jumps at times $t_{k}$. Then, the left- and right-hand sides are measures instead of mere functions, and one may rewrite such dynamics as an equality of measures $m d \dot{q}=f(t) d t+$ $d R$, where $d R$ is an atomic measure with atoms at $t_{k}$. In this case, writing the dynamics as $m(d \dot{q} / d t)(t)=\cdots$ is meaningless because at a jump time $d \dot{q}$ has no density with respect to the Lebesgue measure $d t$ (it has a density with respect to the Dirac measure $\delta_{t_{k}}$ which in turn is singular to $d t$ ). Now if the right-hand side is a multivalued function $F(t)$, one may write the dynamics as $m \ddot{q}(t) \in F(t)$. The concept of MDI merges both equality of measures and inclusion and has been coined by Moreau [70] and Schatzman [90]. This is an evolution problem of the form $(d x / d \mu)+g\left(x\left(t^{+}\right), t^{+}\right)(d t / d \mu) \in F\left(x\left(t^{+}\right), t^{+}\right)$, where $\mu$ is a positive measure and $F(x, t)$ is a cone, $g(x(t), t)$ is Lebesgue integrable, and the inclusion holds $\mu$ almost everywhere. The notation $d x / d \mu$ generalizes that of a derivative, for measures. For instance if $d \mu=\delta_{t}$ and if $x(\cdot)$ jumps at $t=t_{k}$, then $(d x / d \mu)\left(t_{k}\right)=x\left(t_{k}^{+}\right)-x\left(t_{k}^{-}\right)$. The fact that $d \mu$ can be any positive measure comes from the fact that $F(x, t)$ is a cone. If $F(x, t)$ is merely convex this no longer holds. If $\dot{q}$ is discontinuous and $q$ is continuous at $t_{k}, k \geq 0$, one may choose $d \mu=d t+\sum_{k \geq 0} \delta_{t_{k}}$ and write the dynamics as $m(d \dot{q} / d \mu)-f(t)(d t / d \mu) \in F(q, t)$. At $t=t_{k}$ one has $d \dot{q} / d \mu=\alpha\left[\dot{q}\left(t_{k}^{+}\right)-\dot{q}\left(t_{k}^{-}\right)\right]$for some $\alpha>0$ whereas $d t / d \mu=$ $d q / d \mu=0$. Outside impacts $d t / d \mu=1$ and $d \dot{q} / d \mu=\ddot{q}(t)$. Finally the functions are evaluated at their right limits because the solutions of Moreau's MDI are right continuous.

\section{APPENDIX B \\ COMPLEMENTARY PROBLEMS (CONTINUED)}

Solving the quadratic program $\min (1 / 2) z^{T} D z+C z, D=$ $D^{T}>0$, subject to $E z+F \geq 0$, is equivalent [74, Sec. 9.3.1] to solving a LCP with $A=E D^{-1} E^{T}, B=-F+E D^{-1} C^{T}$. This has important practical consequences, e.g., for numerical calculations [15], [21], [72]. An ELCP is a complementarity problem that can be written as $\sum_{j=1}^{m} \Pi_{i \in \phi_{j}}(A z-c)_{i}=0, A z-c \geq 0$, $B z=d$, for some matrices $A \in \mathbb{R}^{p \times n}, B \in \mathbb{R}^{q \times n}$, vectors $c \in \mathbb{R}^{p}, d \in \mathbb{R}^{q}$, and $m$ subsets $\phi_{j}$ of $\{1,2, \ldots, p\} ; z_{i}$ denotes 
the $i$ th component of the vector $z$. Many other types of complementarity problems exist [92] which cannot be described here for the sake of briefness of the presentation. A generalized equation is an equation of the form $0 \in F(x)$, where $F(x)$ is a multivalued mapping [79] [i.e., $F(x)=y_{1}$ and $F(x)=y_{2}$ does not imply $y_{1}=y_{2}$; the mappings whose graphs are in Fig. 3(d), (e), (f), (g) and (h) are multivalued]. In particular the complementarity condition $0 \leq x \perp y \geq 0$ can equivalently be rewritten as the generalized equation $0 \in x+\partial \psi_{\left(\mathbf{R}^{+}\right)^{m}}(y)$, which also shows the link between complementarity and convex analysis [86, p. 226]. This will also be useful to transform complementarity systems into various sorts of differential inclusions.

$\Longrightarrow$ Therefore, one sees that there are strong links between generalized equations, convex analysis, and complementarity problems.

\section{ACKNOWLEDGMENT}

The author would like to thank M. Monteiro-Marques, M. Heemels, M. Mabrouk, and M. di Bernardo for some fruitful discussions on parts of this paper and preliminary versions.

\section{REFERENCES}

[1] M. Abadie, "Dynamic simulation of rigid bodies: Modeling of frictional contact," in Impacts in Mechanical Systems. Analysis and Modeling. ser. Lecture Notes in Physics LNP 551, B. Brogliato, Ed. Berlin, Germany: Springer-Verlag, 2000, pp. 61-144.

[2] P. Alart, "Méthode de Newton généralisée en mécanique du contact," $J$. Math. Pure. Appl., vol. 76, pp. 93-108, 1997.

[3] Automatica, vol. 35, no. 3, Mar. 1999.

[4] D. D. Bainov and P. S. Simeonov, Systems With Impulse Effects; Stability, Theory and Applications. New York: Wiley, 1989.

[5] P. Ballard, "The dynamics of discrete mechanical systems with perfect unilateral constraints," Arch. Rational Mech. Anal., vol. 154, no. 3, pp. 199-274, 2000

[6] A. Bemporad, G. Ferrari-Trecate, and M. Morari, "Observability and controllability of piecewise affine and hybrid systems," IEEE Trans. Automat. Contr., vol. 45, pp. 1854-1864, Oct. 2000.

[7] A. Bemporad, W. P. M. H. Heemels, and B. de Schutter, "On hybrid systems and closed-loop MPC systems," IEEE Trans. Automat. Contr., vol. 47, pp. 863-869, May 2002.

[8] S. C. Billups and K. G. Murty, "Complementarity problems," J. Comput. Appl. Math., vol. 124, pp. 303-318, 2000.

[9] A. Bressan and F. Rampazzo, "On differential systems with quadratic impulses and their application to Lagrangian mechanics," SIAM J. Control Optim., vol. 31, no. 5, pp. 1205-1220, Sept. 1993.

[10] H. Brezis, Opérateurs Maximaux Monotones. Amsterdam, The Netherlands: North-Holland, 1973.

[11] B. Brogliato, S. Niculescu, and P. Orhant, "On the control of finite dimensional mechanical systems with unilateral constraints," IEEE Trans. Automat. Contr., vol. 42, pp. 200-215, Feb. 1997.

[12] B. Brogliato, Nonsmooth Mechanics, 2nd ed. London, U.K.: SpringerVerlag, 1999.

[13] B. Brogliato, S. I. Niculescu, and M. D. P. Monteiro Marques, "On tracking control of a class of complementarity-slackness mechanical systems," Syst. Control Lett., vol. 39, no. 4, pp. 255-266, Mar. 2000.

[14] B. Brogliato, Ed., Impacts in Mechanical Systems. Analysis and Modeling. ser. Lecture Notes in Physics 551. Berlin, Germany: SpringerVerlag, 2000.

[15] B. Brogliato, A. A. ten Dam, L. Paoli, F. Génot, and M. Abadie, "Numerical simulation of finite dimensional multibody nonsmooth mechanical systems," ASME Appl. Mech. Rev., vol. 55, no. 2, pp. 107-150, Mar. 2002.

[16] B. Brogliato and A. Zavala-Rio, "On the control of complementarityslackness juggling mechanical systems," IEEE Trans. Automat. Contr., vol. 45, pp. 235-246, Feb. 2000.

[17] B. Brogliato, M. Mabrouk, and A. Zavala-Rio, "A controllability criterion for linear juggling mechanical systems," presented at the 3rd Contact Mechanics Int. Symp., Peniche, Portugal, June 17-21, 2001, INRIA Rep. 6772.
[18] B. Brogliato, "Absolute stability and the Lagrange-Dirichlet theorem with monotone multivalued mappings," presented at the 40th IEEE Conf. Decision Control, Orlando, FL, Dec. 4-7, 2001, INRIA Rep. 6794.

[19] F. Bullo and M. Zefran, "Modeling and controllability for a class of hybrid mechanical systems," IEEE Trans. Robot. Automat., vol. 18, pp. 563-573, Apr. 2002.

[20] M. K. Çamlibel, W. P. M. H. Heemels, and J. M. Schumacher, "On linear passive complementarity systems," Eur. J. Control, vol. 8, no. 3, pp. 220-237, 2002.

[21] M. K. Çamlibel, "Complementarity methods in the analysis of piecewise linear dynamical systems," Ph.D. dissertation, Tilburg Univ., The Netherlands, 2001.

[22] M. K. Çamlibel and J. M. Schumacher, "Existence and uniqueness of solutions for a class of piecewise linear dynamical systems," Linear Alg. Applicat., vol. 351-352, pp. 147-184, 2002.

[23] F. H. Clarke, "Optimization and nonsmooth analysis," SIAM Classics Appl. Math., p. 5, 1990.

[24] J. Cortès, Geometric, Control, and Numerical Aspects of Nonholonomic Systems, ser. Lecture Notes in Mathematics 1793, New York: SpringerVerlag, 2002.

[25] A. Curnier, "Unilateral contact: Mechanical modeling," in New Developments in Contact Problems, P. Wriggers and P. Panagiotopoulos, Eds. New York: Springer-Verlag, 1999, pp. 1-54.

[26] A. A. ten Dam, E. Dwarshuis, and J. C. Willems, "The contact problem for linear continuous-time dynamical systems: A geometric approach," IEEE Trans. Automat. Contr., vol. 42, pp. 458-472, Apr. 1997.

[27] H. Dankowicz and P. T. Piiroinen, "Exploiting discontinuities for stabilization of recurrent motions," Dyna. Syst. Int. J., 2003, to be published.

[28] K. Deimling, "Multivalued differential equations," in De Gruyter Series in Nonlinear Analysis and Applications. Berlin, Germany: SpringerVerlag, 1992.

[29] J. Dieudonné, Eléments d'Analyse, tome 2 Gauthier-Villars, Paris, 1969.

[30] E. Dimnet and M. Frémond, "Collisions involving solids and fluids," in Novel Approaches in Civil Engineering, M. Frémond and F. Macceri, Eds. New York: Springer-Verlag, 2001.

[31] Euro. J. Control, vol. 7, no. 4, 2001.

[32] M. C. Ferris and J. S. Pang, "Engineering and Economic Applications of Complementarity Problems," SIAM Rev., vol. 39, no. 4, pp. 669-713, Dec. 1997.

[33] M. Frémond, Non-Smooth Thermomechanics. Berlin, Germany: Springer-Verlag, 2002.

[34] F. Génot and B. Brogliato, "New results on Painlevé paradoxes," Eur. J. Mech. A/Solids, vol. 18, no. 4, pp. 653-678, July-Aug. 1999.

[35] Ch. Glocker, Set Valued Force Laws, ser. Lecture Notes in Applied Mechanics, LNAM 1. New York: Springer-Verlag, 2001.

[36] D. Goeleven, G. E. Stavroulakis, G. Salmon, and P. D. Panagiotopoulos, "Solvability theory and projection method for a class of singular variational inequalities: Elastostatic unilateral contact applications," $J$. Optim. Theory Applicat., vol. 95, no. 2, 1996.

[37] D. Goeleven, D. Motreanu, Y. Dumont, and M. Rochdi, Variational and Hemivariational Inequalities. Norwell, MA: Kluwer, 2002.

[38] B. Goodwine and J. W. Burdick, "Controllability of kinematic control systems on stratified configuration spaces," IEEE Trans. Automat. Contr., vol. 46, pp. 358-368, Mar. 2001.

[39] B. Halphen and N. Q. Son, "Sur les matériaux généralisés," J. Mécanique, vol. 14, no. 1, pp. 39-63, 1975.

[40] W. P. M. H. Heemels, J. M. Schumacher, and S. Weiland, "The rational complementarity problem," Lin. Alg. Applicat., vol. 294, pp. 93-135, 1999 .

[41] W. P. M. H. Heemels, "Linear complementarity systems. A study in hybrid dynamics," Ph.D. dissertation, Eindhoven Univ. Technol., Eindhoven, The Netherlands, 1999.

[42] W. P. M. H. Heemels, J. M. Schumacher, and S. Weiland, "Linear complementarity systems," SIAM J. Appl. Math., vol. 60, no. 4, pp. 1234-1269, 2000.

[43] W. P. M. H. Heemels, B. De Schutter, and A. Bemporad, "Equivalence of hybrid dynamical models," Automatica, vol. 37, no. 7, pp. 1085-1092, 2001.

[44] J. B. Hiriart-Urruty and C. Lemaréchal, Fundamentals of Convex Analysis. Berlin, Germany: Springer-Verlag, 2001.

[45] H. P. Huang and N. H. McClamroch, "Time optimal control for a robotic contour following problem," IEEE Trans. Robot. Automat., vol. 4, pp. 140-149, Feb. 1988.

[46] Y. Hurmuzlu, F. Génot, and B. Brogliato, "Modeling, stability and control of biped robots-A general framework," Automatica, Oct. 2001, to be published.

[47] IEEE Trans. Automat. Contr., vol. 43, Apr. 1998.

[48] Int. J. Robust Nonlinear Control, vol. 11, no. 5, 2001. 
[49] H. de Jong, "Modeling and simulation of genetic regulatory systems: A literature survey," J. Computat. Biol., vol. 9, no. 1, pp. 67-103, 2002

[50] N. Kenmochi and I. Pawlow, "A class of nonlinear elliptic-parabolic equations with time-dependent constraints," Nonlinear Anal. TMA, vol. 10, pp. 1181-1202, 1986.

[51] V. V. Kozlov and D. V. Treshchev, Billiards: A Genetic Introduction to the Dynamics of Systems With Impacts. Providence, RI: Amer. Math. Soc., 1991.

[52] M. Krupa, A. Khun, W. Poth, M. Schagerl, W. Steiner, A. Steindl, H Troger, and G. Wiedermann, "Tethered satellite systems: A new concept of space flight," Eur. J. Mech. A/Solids, vol. 19, pp. S149-S164, 2000.

[53] M. Kunze and M. Monteiro Marques, "An introduction to Moreau's sweeping process," in Impacts in Mechanical Systems, B. Brogliato, Ed. New York: Springer-Verlag, 2000, pp. 1-60.

[54] A. D. Lewis and R. M. Murray, "Configuration controllability of simple mechanical control systems," SIAM J. Control Optim., vol. 35, no. 3, pp. 766-790, 1997.

[55] R. Leine, B. Brogliato, and H. Nijmeijer, "Periodic motion and bifurcations induced by the Painlevé paradox," Eur. J. Mech. A/Solids, vol. 21, pp. 869-896, 2002

[56] H. L. Lin and M. Zacksenhouse, "Yoyo dynamics: Sequence of collisions captured by a restitution effect," ASME J. Dyna. Syst., Measure., Control, vol. 124, no. 3, pp. 390-397, Sept. 2002.

[57] R. Lozano, B. Brogliato, O. Egeland, and B. Maschke, Dissipative Systems Analysis and Control. Theory and Applications. London, U.K.: Springer-Verlag, 2000.

[58] K. M. Lynch and C. K. Black, "Recurrence, controllability, and stabilization of juggling," IEEE Trans. Robot. Automat., vol. 17, no. 21, pp. 113-124, Apr. 2001.

[59] M. Mabrouk, "A unified variational model for the dynamics of perfect unilateral constraints," Euro. J. Mech. A/Solids, vol. 17, no. 5, pp. 819-842, 1998

[60] M. Mata Jimenez and B. Brogliato, "Analysis of PD and nonlinear control of mechanical systems with dynamic backlash," J. Vibrat. Control, vol. 9, no. 1, pp. 119-156, 2003.

[61] L. Menini and A. Tornambe, "State estimation of (otherwise unobservable) linear mechanical systems through the use of nonsmooth impacts: The case of two mating gears," Automatica, vol. 38, no. 10, pp. 1823-1826, 2002.

[62] L. Menini and A. Tornambe, "Asymptotic tracking of periodic trajectories for a simple mechanical system subject to nonsmooth impacts," IEEE Trans. Automat. Contr., vol. 46, pp. 1122-1126, July 2001.

[63] M. D. P. Monteiro-Marques, Differential Inclusions in Nonsmooth Mechanical Problems: Shocks and Dry Friction. Boston, MA Birkhauser, 1993, PNLDE 9.

[64] J. J. Moreau, Les liaisons unilatérales et le principe de Gauss. Paris, France: CRAS Paris, 1963, vol. 256, pp. 871-874.

[65] — - "Fonctionnelles convexes," in Séminaire sur les Équations aux Dérivées Partielles. Paris, France: Collége de France, 1966-1967.

[66] - La Notion de Sur-Potentiel et les Liaisons Unilaétrales en Élastostatique. Paris, France: CRAS Paris, 1968, vol. 271A, pp. 954-957.

[67] _ - "On unilateral constraints, friction and plasticity," in New Variational Techniques in Mathematical Physics, CIME II ciclo 1973, G. Capriz and G. Stampacchia, Eds, Italy: Edizioni Cremonese, 1974, pp. $175-322$.

[68] — "Evolution problem associated with a moving convex set in a Hilbert space," J. Diff. Equat., vol. 26, pp. 347-374, 1977.

[69] —- "Application of convex analysis to some problems of dry friction," in Trends in Applications of Pure Mathematics to Mechanics, H. Zorski, Ed. London, U.K.: Pitman, 1979, vol. 2, pp. 263-280.

[70] - Liaisons Unilatérales Sans Frottement et Chocs Inélastiques, ser. II. Paris, France: C.R. Acad. Sc. Paris t.296, 1983, pp. 1473-1476.

[71] _ "Unilateral contact and dry friction in finite freedom dynamics," in CISM Courses and Lectures no 302, International Centre for Mechanical Sciences, J. J. Moreau and P. D. Pnagiotopoulos, Eds. New York: Springer-Verlag, 1988.

[72] - "Some numerical methods in multibody dynamics: Application to granular materials," Eur. J. Mech. A/Solids, vol. 13, no. 4, pp. 93-114, 1994.

[73] — "An introduction to unilateral dynamics," in Novel Approaches in Civil Engineering, M. Frémond and F. Macceri, Eds. New York: Springer-Verlag, 2003, pp. 1-65.

[74] K. G. Murty. (1997) Linear complementarity, linear and nonlinear programming. [Online]. Available: http://www-personal.engin.umich.edu/ murty/book/LCPbook/

[75] A. Nagurney and D. Zhang, Projected Dynamical Systems and Variational Inequalities With Applications. Norwell, MA: Kluwer, 1996.

[76] S. I. Niculescu and B. Brogliato, "Force measurement time-delays and contact instability phenomena," Eur. J. Control, vol. 5, no. 2-4, pp. 279-292, 1999.
[77] H. Nijmeijer and A. J. van der Shaft, Nonlinear Dynamical Control Systems. New York: Springer-Verlag, 1990.

[78] Yu. Orlov, "Instantaneous impulse response of nonlinear system," IEEE Trans. Automat. Contr., vol. 45, pp. 999-1002, May 2000.

[79] P. D. Panagiotopoulos, Inequality Problems in Mechanics and Applications. Boston, MA: Birkhauser, 1985.

[80] L. Paoli and M. Schatzman, "Mouvement à un nombre fini de degrés de liberté avec contraintes unilatérales: Cas avec perte d'énergie," Math. Model. Numer. Anal., vol. 27, no. 6, pp. 673-717, 1993.

[81] F. Pfeiffer and Ch. Glocker, Multibody Dynamics With Unilateral Contacts. New York: Wiley, 1996.

[82] F. Pfeifer, Ed., Phil. Trans. Royal Soc. Lond., Series A, 2001, vol. 359.

[83] A. Yu. Pogromsky, W. P. M. H. Heemels, and H. Nijmeijer, "On wellposedness of relay systems," presented at the IFAC Symp. NOLCOS, 2001.

[84] P. J. Rabier and W. C. Rheinboldt, "On the computation of impasse points of quasilinear differential-algebraic equations," Math. Comput., vol. 62, no. 205, pp. 133-154, Jan. 1994.

[85] N. Ramachandran, S. S. Pande, and N. Ramakrishnan, "The role of deburring in manufacturing: A state-of-the-art survey," J. Mater. Processing, vol. 44, pp. 1-13, 1994.

[86] R. T. Rockafellar, Convex Analysis. Princeton, NJ: Princeton Univ. Press, 1972.

[87] A. van der Schaft and H. Schumacher, An Introduction to Hybrid Dynamical Systems. London, U.K.: Springer-Verlag, 2000, LNCIS 251.

[88] A. van der Schaft and J. M. Schumacher, "Complementarity modeling of hybrid systems," IEEE Trans. Automat. Contr., vol. 43, pp. 483-490, Apr. 1998.

[89] - "The complementarity-slackness class of hybrid systems," Math. Control, Signals, Syst., vol. 9, pp. 266-301, 1996.

[90] M. Schatzman, "A class of nonlinear differential equations of second order in time," Nonlinear Anal., Theory, Meth., Applicat., vol. 2, no. 3 , pp. $355-373,1978$.

[91] W. W. Schmaedeke, "Optimal control theory for nonlinear vector differential equations containing mesures," SIAM J. Control, ser. A, vol. 3, no. 2, pp. 231-280, 1965.

[92] B. de Schutter and B. de Moor, "The linear dynamic complementarity problem is a special case of the extended linear complementarity problem," Syst. Control Lett., vol. 34, pp. 63-75, 1998.

[93] E. D. Sontag, Mathematical Control Theory. New York: SpringerVerlag, 1990, vol. TAM 6.

[94] M. W. Spong, "Impact controllability of an air hockey puck," Syst. Control Lett., vol. 42, no. 5, pp. 333-345, 2001.

[95] D. E. Stewart, "Rigid-body dynamics with friction and impacts," SIAM Rev., vol. 42, no. 1, pp. 3-39, 2000

[96] W. J. Stronge, Impact Mechanics. Cambridge, U.K.: Cambridge Univ. Press, 2000.

[97] Syst. Control Lett., vol. 38, no. 3, 1999

[98] G. Tao, X. Ma, and Y. Ling, "Optimal and nonlinear decoupling control of systems with sandwiched backlash," Automatica, vol. 37, no. 2, pp. 165-176, Feb. 2001.

[99] L. Vandenberghe, B. L. de Moor, and J. Vandewalle, "The generalized linear complementarity problem applied to the complete analysis of resistive piecewise-linear circuits," IEEE Trans. Circuits Syst., vol. 36, pp. 1382-1391, Nov. 1989.

[100] J. Warga, "Minimizing variational curves restricted to a preassigned set," Trans. Amer. Math. Soc., vol. 112, pp. 432-455, 1964.

[101] J. M. Bourgeot and B. Brogliato, "Tracking control of complementarity Lagrangian systems," Int. J. Bifurcation Chaos, Spec. Iss. "Non-smooth Dymanical Systems: Recent Trends and Perspectives", 2003, to be published. 